\title{
OH (1720 MHz) Masers: A Multiwavelength Study of the Interaction between the W51C Supernova Remnant and the W51B Star Forming Region
}

\author{
C. L. Brogan ${ }^{1}$, W. M. Goss², T. R. Hunter ${ }^{1}$, A. M. S. Richards ${ }^{3}$, C. J. Chandler ${ }^{2}$, J. S. \\ Lazendic $^{4}$, B.-C. Koo ${ }^{5}$, I. M. Hoffman ${ }^{6}$, \& M. J. Claussen ${ }^{2}$
}

\begin{abstract}
We present a comprehensive view of the W51B H II region complex and the W51C supernova remnant using new radio observations from the VLA, VLBA, MERLIN, JCMT, and CSO along with archival data from Spitzer, ROSAT, $A S C A$, and Chandra. Our VLA data include the first $\lambda=400 \mathrm{~cm}(74 \mathrm{MHz})$ continuum image of W51 at high resolution $\left(88^{\prime \prime}\right)$. The $400 \mathrm{~cm}$ image shows nonthermal emission surrounding the G49.2-0.3 H II region, and a compact source of non-thermal emission (W51B_NT) coincident with the previously-identified $\mathrm{OH}$ $(1720 \mathrm{MHz})$ maser spots, non-thermal 21 and $90 \mathrm{~cm}$ emission, and a hard Xray source. W51B_NT falls within the region of high likelihood for the position of $\mathrm{TeV} \gamma$-ray emission. Using the VLBA three $\mathrm{OH}(1720 \mathrm{MHz})$ maser spots are detected in the vicinity of W51B_NT with sizes of 60 to 300 AU and Zeeman effect magnetic field strengths of 1.5 to $2.2 \mathrm{mG}$. The multiwavelength data demonstrate that the northern end of the W51B H II region complex has been partly enveloped by the advancing W51C SNR and this interaction explains the presence of W51B_NT and the $\mathrm{OH}$ masers. This interaction also appears in the thermal molecular gas which partially encircles W51B_NT and exhibits narrow pre-shock $\left(\Delta v \sim 5 \mathrm{~km} \mathrm{~s}^{-1}\right)$ and broad post-shock $\left(\Delta v \sim 20 \mathrm{~km} \mathrm{~s}^{-1}\right)$ velocity components. RADEX radiative transfer modeling of these two components
\end{abstract}

\footnotetext{
${ }^{1}$ National Radio Astronomy Observatory, 520 Edgemont Rd, Charlottesville, VA 22903, USA; cbrogan@nrao.edu.

${ }^{2}$ National Radio Astronomy Observatory, P. O. Box 0, Socorro, NM 87801, USA

${ }^{3}$ Jodrell Bank Centre for Astrophysics, Turing Building, University of Manchester, Manchester M13 9PL, $\mathrm{UK}$

${ }^{4}$ Monash Unversity, Clayton, VIC 3800, Australia

${ }^{5}$ Astronomy Program, SEES, Seoul National University, Seoul 151-742, South Korea

${ }^{6}$ Wittenberg University, Springfield, OH 45501, USA
} 
yield physical conditions consistent with the passage of a non-dissociative Ctype shock. Confirmation of the W51B/W51C interaction provides additional evidence in favor of this region being one of the best candidates for hadronic particle acceleration known thus far.

Subject headings: ISM: individual(W51B) — ISM: individual(W51C) — supernova remnants - H II regions - masers - ISM: molecules

\section{INTRODUCTION}

Star formation triggered by the interaction of supernova remnants (SNRs) with their parent molecular clouds has long been thought to play an important role in the production of new generations of stars. Indeed, it is thought that the enrichment of our own Solar System with heavy elements might have resulted from such an interaction. In practice such interactions are very difficult to observe due to the extreme kinematic complexity present toward the inner Galactic plane where most massive stars and SNRs are found. Over the last two decades it has been recognized that when $\mathrm{OH}(1720 \mathrm{MHz})$ masers are found toward SNRs they act as signposts for SNR/molecular cloud interactions (see for example Wardle \& Yusef-Zadeh 2002; Frail et al. 1996; Green et al. 1997; Frail \& Mitchell 1998; Frail 2011). To date, OH $(1720 \mathrm{MHz})$ masers have been found in $\sim 24$ SNRs, or $10 \%$ of the known SNRs in our Galaxy (Frail et al. 1996; Yusef-Zadeh et al. 1996; Green et al. 1997; Koralesky et al. 1998; Sjouwerman \& Pihlström 2008; Hewitt \& Yusef-Zadeh 2009). Indeed, all $24 \mathrm{OH}(1720 \mathrm{MHz})$ maser SNRs can be found in the Jiang et al. (2010) catalogue of 34 SNRs thought to be interacting with associated molecular clouds.

The observational properties of these SNR masers are quite different from those of H II region $\mathrm{OH}(1720 \mathrm{MHz})$ masers. SNR OH (1720 MHz) masers have: (1) larger maser spot sizes; (2) narrow and simple line profiles; (3) low levels of circular polarization (typically $<10 \%$ ); (4) low magnetic field strengths $(<3 \mathrm{mG})$; (5) relatively non-variable flux densities; (6) a notable lack of any other maser species; and (7) low luminosity (Yusef-Zadeh et al. 1996; Claussen et al. 1997, 1999.b, 2002; Koraleskv et al. 1998; Brogan et al.|2000; Hoffman et al. 2003, 2005a, b; Woodall \& Gray 2007; McDonnell et al. 2008; Lazendic et al. 2010; Pihlström et al. 2011). These observational facts can be explained if $\mathrm{OH}(1720 \mathrm{MHz}) \mathrm{SNR}$ masers originate in the post-shock molecular gas behind C-type shocks, and are collisionally pumped in contrast to their radiatively pumped H II region counterparts (Elitzur 1976).

Current theories further suggest that the collisional pump is most efficient for post-shock densities of $\sim 1 \times 10^{5} \mathrm{~cm}^{-3}$ and temperatures in the range $50 \mathrm{~K} \lesssim T \lesssim 125 \mathrm{~K}$, and that the 
abundance of $\mathrm{OH}$ must be enhanced by the dissociation of water due to X-rays and/or $\gamma$-rays emanating from the SNR (Lockett et al. 1999; Wardle 1999; Hewitt et al. 2008). Observations of the physical conditions in the pre- and post-shock gas available to date are in good agreement with these theoretical expectations (see e.g. Frail \& Mitchell 1998; Reach et al. 2005). Prior to this work, however, there have been few in-depth studies employing multitransition, multi-species analyses. Interestingly, a number of recent publications have also noted the growing number of $\gamma$-ray detected SNRs that are interacting with nearby molecular clouds. (Hewitt et al. 2009; Castro \& Slane 2010; Méhault 2011; Frail 2011; Uchivama et al. 2012, to name a few). Indeed, many of the $\gamma$-ray SNRs are associated with well known $\mathrm{OH}$ $(1720 \mathrm{MHz})$ sources: e.g. G349.7+0.2,CTB 37A, 3C391, W44, IC443 and W51C. Hence, as a powerful probe of $\mathrm{SNR} /$ molecular cloud interactions, observations of this $\mathrm{OH}$ maser line can potentially pinpoint sites of particle acceleration.

Of the known SNR OH (1720 MHz) masers, the origin of those observed toward the W51 complex remain among the most poorly understood (Green et al. 1997; Brogan et al. 2000). W51 is composed of two large H II region complexes W51A (also known as W51 Main) and W51B, as well as the SNR W51C (see the 90 and $400 \mathrm{~cm}$ images in Figure 1a,b). W51 is located near the Sagittarius arm tangent point at $\sim l=49^{\circ}$ and $\sim b=0.3$ and thus velocity crowding in this direction has made distance determinations (and relative orientations along the line of sight) for the three W51 components (A, B, and C) uncertain. In the past, their distances were considered to be in the range of 5-7 kpc (Kolpak et al. 2003). A maser parallax distance has recently been measured for W51A of $5.4( \pm 0.3) \mathrm{kpc}$ (Sato et al. 2010). Additionally, the soft X-ray absorption seen toward the W51B region compared to the rest of the W51C SNR suggests that W51B lies in front of W51C (Koo et al. 1995). However, it remains uncertain whether W51A is in front of, co-distant with, or behind W51B. The OH $(1720 \mathrm{MHz})$ masers reported by Green et al. (1997) are located $\sim 2.3^{\prime}$ west of the W51B H II region G49.2-0.3. Subsequent observations of these masers by Brogan et al. (2000) demonstrated that these masers exhibit all the hallmarks of SNR OH (1720 MHz) masers. Like Green et al., these authors conclude that these masers are most likely excited by the interaction between the W51C SNR (the only known SNR in the region) and the molecular gas associated with the W51B H II region.

However, the exact nature of this interaction remains unclear especially since SNR $\mathrm{OH}(1720 \mathrm{MHz})$ maser theory suggests that a sufficient column of $\mathrm{OH}$ with the necessary velocity coherence can only be achieved in shocks that move (more or less) perpendicular to the line of sight (see for example Lockett et al. 1999). It is difficult to reconcile the need for an edge-on shock with the fact that W51B lies in front of the W51C SNR (i.e. it seems that any shock would propagate with a substantial component along the line of sight). This paper describes a multiwavelength investigation of the W51B/C OH $(1720 \mathrm{MHz})$ masers and 

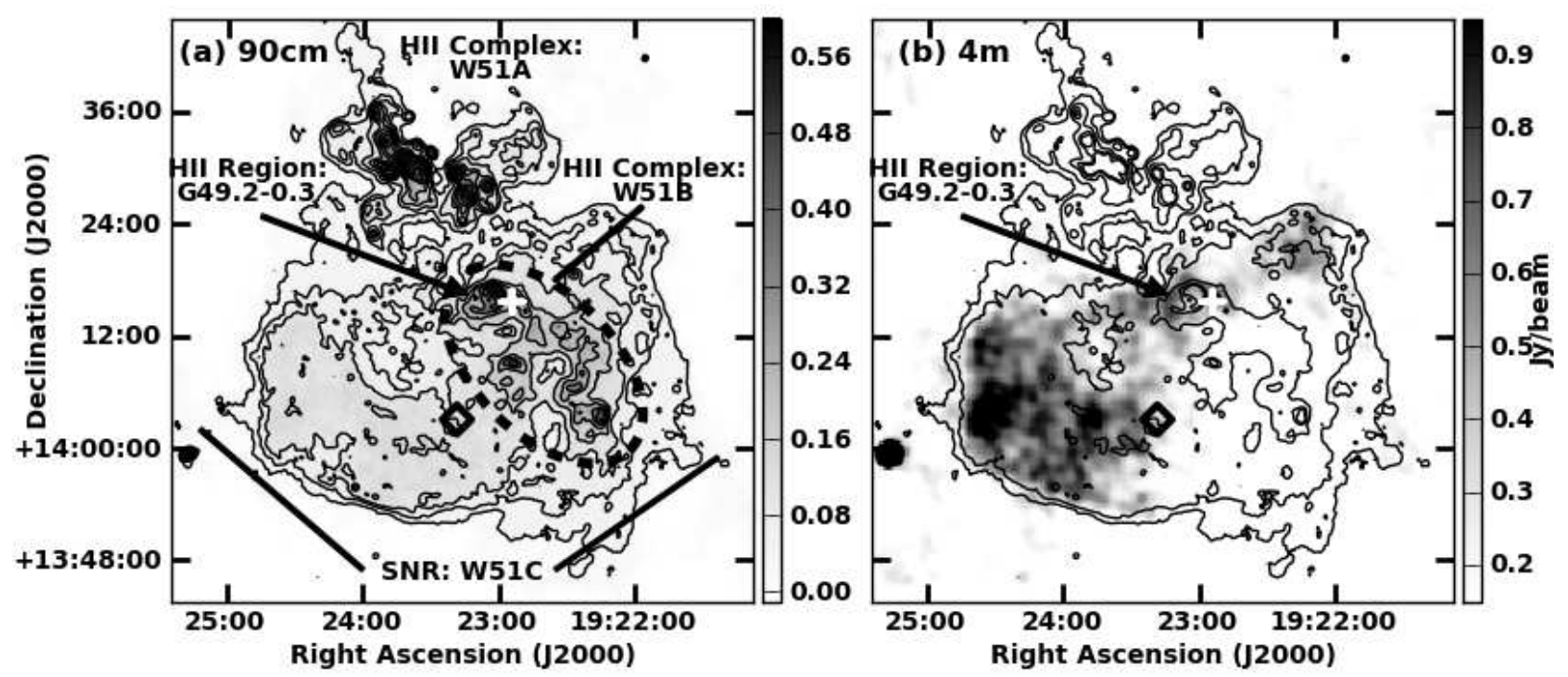

Fig. 1.- (a) VLA $90 \mathrm{~cm} \mathrm{B+C+D}$ configuration image of the W51 complex (greyscale and contours). This image has a resolution of $35^{\prime \prime} \times 33^{\prime \prime}\left(\mathrm{PA}=-86^{\circ}\right)$. The contour levels are $30,60,90,150,210,300,400, \& 500 \mathrm{mJy}^{\text {beam }^{-1}}$. Labels indicate the emission associated with W51A, W51B (dashed ellipse), and W51C.(b) VLA $400 \mathrm{~cm} \mathrm{B+C} \mathrm{configuration} \mathrm{image}$ in greyscale with an angular resolution of $92^{\prime \prime} \times 84^{\prime \prime}\left(\mathrm{PA}=29.8^{\circ}\right)$, with $90 \mathrm{~cm}$ contour levels of $30,60,150,300, \& 400 \mathrm{mJy}^{\text {beam }}{ }^{-1}$. On both panels, the white + symbol shows the location of the $\mathrm{OH}(1720 \mathrm{MHz})$ masers, and the black diamond symbol shows the location of the PWN candidate CXO J192318.5+1403035 from Koo et al. (2005). The W51B H II region G49.2-0.3 is also labeled. 
their environment spanning ten decades in wavelength (400 $\mathrm{cm}$ to X-rays) and six decades of angular resolution (10 mas to $1^{\circ}$ ). The goal of this study is to better understand the properties of the maser emission through high resolution MERLIN 1 and Very Long Baseline Array 2 (VLBA) observations, as well as to understand the physical conditions that have led to the maser emission using Very Large Array (VLA) $3400 \mathrm{~cm}, 90 \mathrm{~cm}$, and $20 \mathrm{~cm}$ data, James Clerk Maxwell Telescope (JCMT) ${ }_{4}^{4} \mathrm{CO}(3-2)$ and ${ }^{13} \mathrm{CO}(2-1)$ data, and Caltech Submillimeter Observatory (CSO) $5 \mathrm{HCO}^{+}(3-2)$ and $\mathrm{HCN}(3-2)$ data. For comparison, we also make use of archival Spitzer mid-IR $8 \mu \mathrm{m}$ data, as well as Chandra, ROSAT, and ASCA X-ray data.

\section{OBSERVATIONS}

The observing parameters for all of the observations described in this paper are listed in Table 1. Additional details are provided below.

\subsection{MERLIN OH (1720 MHz) Maser Observations}

The $\mathrm{OH}(1720 \mathrm{MHz})$ masers were observed for approximately 18 hours (15 hrs on source) over the course of three days using the six antennas of the MERLIN array (Lovell was not used). The Doppler tracking corrections, in the LSR convention, were applied in the correlator. In addition to right circular and left circular polarization data, the RL and LR cross correlation data were also recorded. Absolute flux and bandpass calibration were carried out using observations of 3C 84. Initial phase calibration was obtained from periodic observations of J1922+1530. Instrumental polarization leakage corrections were derived from the unpolarized source 3C84, and 3C286 was used to set the absolute polarization angle. The data were processed using the AIPS software package.

\footnotetext{
${ }^{1}$ MERLIN is a UK national facility operated by University of Manchester on behalf of STFC.

${ }^{2}$ The National Radio Astronomy Observatory operates the Very Long Baseline Array and is a facility of the National Science Foundation operated under a cooperative agreement by Associated Universities, Inc.

${ }^{3}$ The National Radio Astronomy Observatory operates the Very Large Array and is a facility of the National Science Foundation operated under a cooperative agreement by Associated Universities, Inc.

${ }^{4}$ The James Clerk Maxwell Telescope is operated by The Joint Astronomy Centre on behalf of the Science and Technology Facilities Council of the United Kingdom, the Netherlands Organisation for Scientific Research, and the National Research Council of Canada.

${ }^{5}$ The Caltech Submillimeter Observatory is operated by the California Institute of Technology under cooperative agreement with the National Science Foundation (AST-0838261).
} 
After initial amplitude, delay, and phase calibration, several iterations of self-calibration on the strongest (W51_2) maser channel were carried out and subsequently applied to the full spectral line dataset.

\section{2. $\quad \mathrm{VLBA}+\mathrm{Y} 1 \mathrm{OH}(1720 \mathrm{MHz})$ Maser Observations}

The $\mathrm{OH}(1720 \mathrm{MHz})$ masers were observed for approximately 12 hours (6 hrs on source) over the course of two days using the ten antennas of the VLBA plus one VLA (Y1) antenna. Both right circular and left circular polarization data were recorded. All calibration and imaging for these data were carried out using the AIPS software. Absolute flux calibration was determined from periodic system temperature measurements coupled with existing antenna gain tables. Bandpass and delay calibration were performed using observations of the strong continuum source J2253+1608 (3C 454.3). In order to obtain accurate absolute positions and sensitivity to potentially weak maser emission, these observations were phase referenced. Since no calibration source suitable for VLBA observations was known at that time, a short VLBA survey of near-by VLA calibrators was carried out prior to the target observations. From this survey, the JVAS source J1911+1611 $\left(19^{\mathrm{h}} 11^{\mathrm{m}} 58.2574^{\mathrm{s}},+16^{\circ} 11^{\prime} 46.865^{\prime \prime}\right.$ with an uncertainty of $\sim 1$ mas) had the strongest compact emission and was used to phase reference the maser data with a cycle time of 2 minutes (this source is now available in the VLBA calibrator catalog). Corrections for Doppler tracking of the target data were applied offline in AIPS.

After initial amplitude, delay, and phase calibration, several iterations of self-calibration on the strongest (W51_2a) maser channel were carried out and subsequently applied to the full line dataset.

\subsection{VLA 21, 90, and $400 \mathrm{~cm}$ Continuum Observations}

We have obtained VLA observations from the $\mathrm{B}+\mathrm{C}$ configurations at $400 \mathrm{~cm}$ and $\mathrm{A}+\mathrm{B}+\mathrm{C}+\mathrm{D}$ configurations at $90 \mathrm{~cm}$ (see Table 1 for details). The FWHP primary beam size of the $90 \mathrm{~cm}$ and $400 \mathrm{~cm}$ data are $\sim 3^{\circ}$ and $\sim 11^{\circ}$, respectively. The data were reduced and imaged using the low frequency wide-field imaging techniques described in Brogan et al. (2004, and references therein). Gain, bandpass, and absolute flux calibration for the $90 \mathrm{~cm}$ data were carried out using J1924+334, Cygnus A, and 3C 48 respectively. In order to best match the $400 \mathrm{~cm}$ data a $90 \mathrm{~cm}$ image was constructed using only the $\mathrm{B}+\mathrm{C}+\mathrm{D}$ configurations and the multiscale clean algorithm in AIPS, with scales of $\sim 40^{\prime \prime}$ (i.e. the intrinsic 
beam given the combination of data and weighting), $120^{\prime \prime}$, and $480^{\prime \prime}$. To achieve the highest angular resolution, a $90 \mathrm{~cm}$ image was also constructed from the $\mathrm{A}+\mathrm{B}+\mathrm{C}+\mathrm{D}$ configuration data and multiscale clean with scales of $\sim 20^{\prime \prime}$ (i.e. the intrinsic beam given the combination of data and weighting), 100", $200^{\prime \prime}$, and $300^{\prime \prime}$.

Absolute flux, bandpass, and coarse phase calibration at $400 \mathrm{~cm}$ were all obtained from observations of Cygnus A (located $\sim 28^{\circ}$ distant). Of particular note is the lack of accurate absolute position information for the $400 \mathrm{~cm}$ data due to the large distance to the $400 \mathrm{~cm}$ phase calibrator. Typically this problem is solved by using a $90 \mathrm{~cm}$ image as an initial model for the $400 \mathrm{~cm}$ self-calibration. However, in the case of the W51 region, much of the total $90 \mathrm{~cm}$ flux is dominated by H II regions making it unsuitable for a $400 \mathrm{~cm}$ model $(400 \mathrm{~cm}$ emission is absorbed by ionized thermal emission along the line-of-sight creating distinct differences in their morphology, see Fig. 1a,b). Therefore, the $400 \mathrm{~cm}$ images have been shifted by $+20^{\prime \prime}$ in R.A. and $-10^{\prime \prime}$ in Dec. so that the background point sources detected at both $400 \mathrm{~cm}$ and $90 \mathrm{~cm}$ (throughout the $5^{\circ} 90 \mathrm{~cm}$ primary beam) are coincident.

We have also obtained new VLA C-configuration $21 \mathrm{~cm} \mathrm{H} \mathrm{I} \mathrm{spectral} \mathrm{line} \mathrm{data} \mathrm{toward}$ the W51B region (primary beam $\sim 30^{\prime}$ ). The continuum from the line free-channels of these data have been combined with the continuum from line free-channels of archival VLA Dconfiguration $21 \mathrm{~cm} \mathrm{H}$ I data (Koo 1997) to form a $21 \mathrm{~cm}$ continuum image with a resolution matching that of the $90 \mathrm{~cm} \mathrm{~B}+\mathrm{C}+\mathrm{D}$ configuration data. It should be noted, however, that owing to spatial filtering the $21 \mathrm{~cm}$ data are missing flux on scales larger than $\sim 15^{\prime}$ compared to $\sim 70^{\prime}$ for the $90 \mathrm{~cm}$ data.

\subsection{JCMT CO (3-2) and ${ }^{13} \mathrm{CO}(2-1)$ Observations}

In order to investigate the nature of the shocked molecular gas in the vicinity of the $\mathrm{OH}(1720 \mathrm{MHz})$ masers we observed the $\mathrm{CO}(3-2)$ line using the $345 \mathrm{GHz} \mathrm{B} 3$ receiver at the JCMT. The zenith opacity at $225 \mathrm{GHz}$ measured by the tipping radiometer at the CSO ranged from 0.10-0.12. Data were taken in raster mode with 4-s integrations per spectrum with samples separated by $5^{\prime \prime}$ in right ascension and $7^{\prime \prime}$ in declination. The final raster is $4^{\prime} \times 5^{\prime}$ in extent. Typical system temperatures were between 400-500 K. The data were reduced using the SPECX software package. A linear baseline was subtracted from each spectrum using channels outside of the line emission region. In order to minimize baseline ripples we used a nearby off-position that contained low level $\mathrm{CO}(3-2)$ emission. To account for this emission we also observed the reference position for 10 minutes in frequency switching mode, and added the resulting emission back to each spectrum. In this paper, the $\mathrm{CO}(3-2)$ line intensity is presented in units of $T_{M B}(\mathrm{~K})$, where $T_{M B}=T_{a}^{*} / 0.63$. The final image cube 

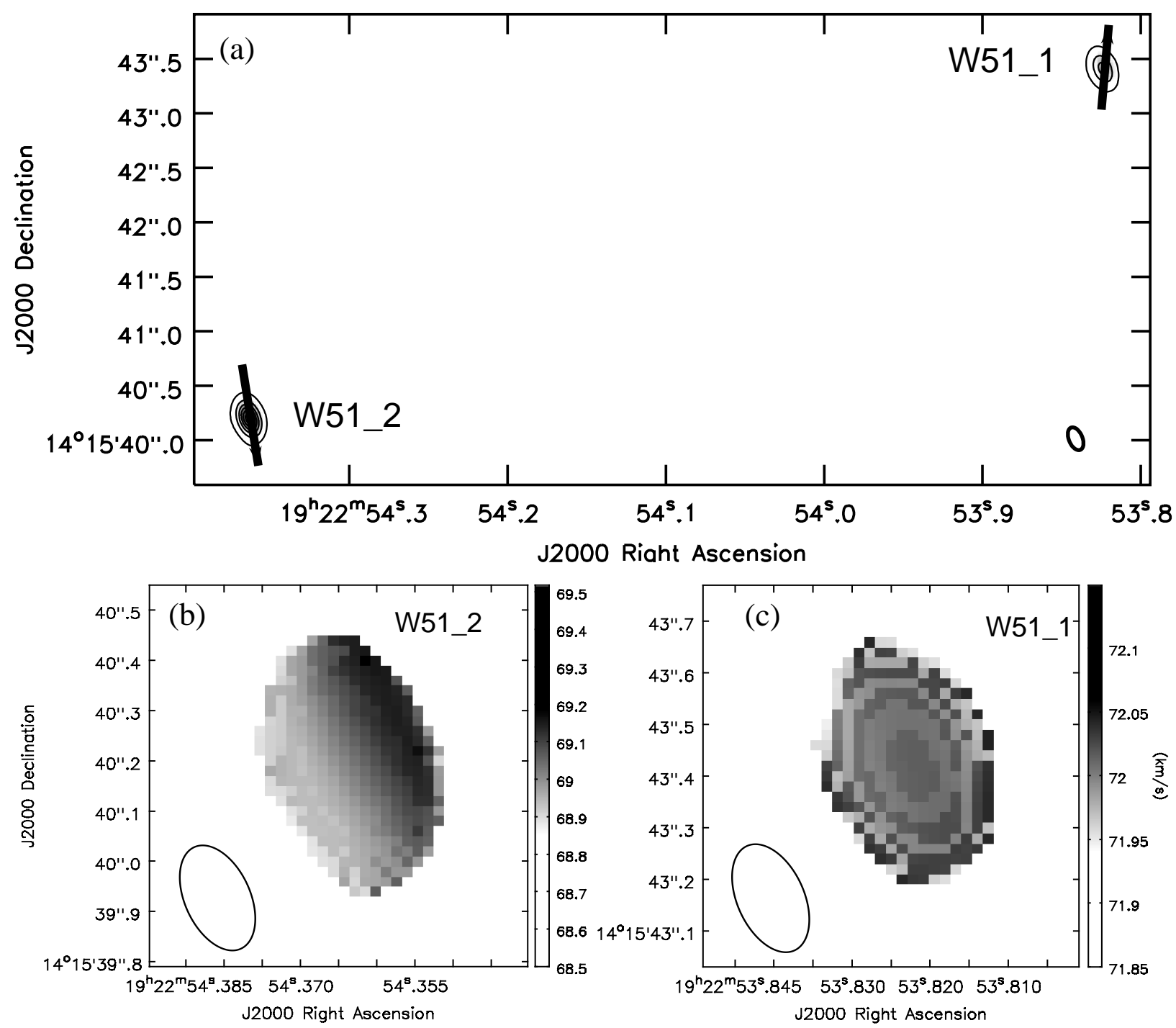

Fig. 2.- (a) MERLIN integrated intensity image (greyscale and contours) of maser spots W51_1 and W51_2. The contour levels are 100, 500, 1000, 1500, \& 2000 mJy beam ${ }^{-1 *} \mathrm{~km} \mathrm{~s}^{-1}$. The beam with a size of $221 \times 125$ mas P.A. $=22.8^{\circ}$ is shown in the lower right. The linear polarization position angle is also shown. (b) Zoomed image of the 1st moment of W51_2 (c) Zoomed image of the 1st moment of W51_1. Note the SE/NW velocity gradient of W51_2, while W51_1 shows no velocity structure. Note that the velocity scales are different in (b) and (c). 
was smoothed to $20^{\prime \prime}$ resolution.

A smaller region $\left(3^{\prime} \times 2^{\prime}\right)$ around the $\mathrm{OH}(1720 \mathrm{MHz})$ masers was also mapped in ${ }^{13} \mathrm{CO}(2-$ 1) using receiver $A 3$ when the zenith opacity was 0.13 . The samples were taken every $7^{\prime \prime}$ in right ascension and every $10^{\prime \prime}$ in declination with 4 -s integrations. The system temperatures ranged from $250-300 \mathrm{~K}$. The angular resolution of these data is $20^{\prime \prime}$. For ${ }^{13} \mathrm{CO}(2-1)$, we used $T_{M B}=T_{a}^{*} / 0.69$.

\section{5. $\mathrm{CSO} \mathrm{HCO}^{+}(3-2)$ and $\mathrm{HCN}(3-2)$ Observations}

We observed the maser position in $\mathrm{HCN}(3-2)$ at $265.886 \mathrm{GHz}$ and $\mathrm{HCO}^{+}(3-2)$ at 267.558 GHz simultaneously using the Caltech Submillimeter Observatory (CSO) in good conditions $\left(\tau_{225 \mathrm{GHz}}=0.05\right)$. At these frequencies, the beam size is $26^{\prime \prime}$ and the main beam efficiency is 0.7 . Using position-switched mode, we acquired 11 minutes of on source integration using the $230 \mathrm{GHz}$ receiver and the facility FFTS autocorrelators. The reference position was $19^{\mathrm{h}} 22^{\mathrm{m}} 44^{\mathrm{s}} .30,+14^{\circ} 05^{\prime} 50^{\prime \prime}$. (J2000) (Ceccarelli et al. 2011). The data were processed using the CLASS software package. The final velocity resolution was smoothed to $0.60 \mathrm{~km} \mathrm{~s}^{-1}$ for $\mathrm{HCO}^{+}$and $1.2 \mathrm{~km} \mathrm{~s}^{-1}$ for $\mathrm{HCN}$ to improve the signal-to-noise. The results are presented in $T_{M B}=T_{a}^{*} / 0.70$.

\section{RESULTS}

\subsection{MERLIN and VLBA OH (1720 MHz) Data}

\subsubsection{Maser Spectral Line Properties}

Figure 2a shows the MERLIN integrated intensity of the two $\mathrm{OH}(1720 \mathrm{MHz})$ masers spots detected toward W51B. The locations of the maser spots with respect to the W51 region is shown in Fig. 1. The positions and central velocities are in good agreement with those reported by Frail et al. (1996); Green et al. (1997); Brogan et al. (2000) from VLA observations (see Table 2). The results from fitting a Gaussian profile to the maser spectra at the emission peaks are given in Table 2, along with the results from earlier VLA observations

for reference (Brogan et al. 2000). At the resolution of the MERLIN data $(221 \times 125$ mas, P.A. $=22.8^{\circ}$ ) the two maser spots remain unresolved. However, maps of the velocity field of the two masers (see Figs. 2b, and c) are notably different, with the W51_1 spot showing a moire pattern indicative of no resolved velocity structure (note the very small width of the displayed velocities), while the W51_2 spot shows a clear SE-NW velocity gradient with 

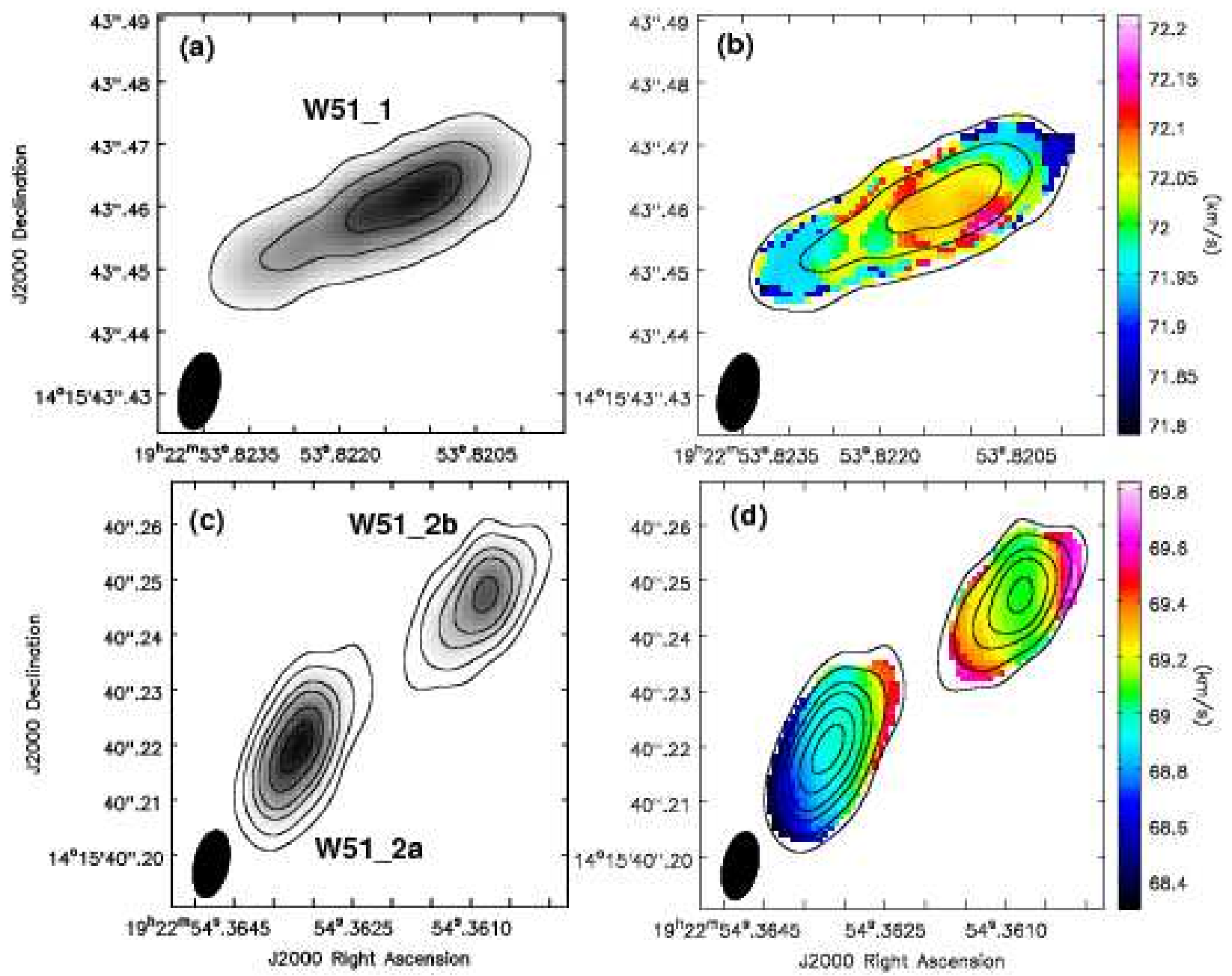

Fig. 3.- (a) VLBA integrated intensity image (greyscale and contours) of maser spot W51_1. The contour levels are $50(3 \sigma), 100, \& 150 \mathrm{mJy}_{\text {beam }}^{-1 *} \mathrm{~km} \mathrm{~s}^{-1}$. (b) Color image of the 1st moment (velocity gradients) superposed with the contours from (a). (c) VLBA integrated intensity image (greyscale and contours) of maser spot W51_2. The contour levels are $50(3 \sigma), 100,200,300,500,700, \& 900 \mathrm{mJy}_{\text {beam }}{ }^{-1 *} \mathrm{~km} \mathrm{~s}^{-1}$. (d) Colorscale of the 1st moment (velocity gradients) superposed with the contours from (c). For all four plots the beam size of $12.5 \times 6.3$ mas P.A. $=-5.2^{\circ}$ is shown in the lower left. 
higher velocities to the NW. The fitted MERLIN peak velocity and FWHM line width is $72.018 \pm 0.002 \mathrm{~km} \mathrm{~s}^{-1}$ and $0.902 \pm 0.004 \mathrm{~km} \mathrm{~s}^{-1}$ for W51_1, while for W51_2 the fitted values are $69.031 \pm 0.001$ and $1.283 \pm 0.002 \mathrm{~km} \mathrm{~s}^{-1}$ (also see Table 21). For comparison, at assumed kinetic temperatures of 50 to $125 \mathrm{~K}$, the thermal line width of $\mathrm{OH}$ ranges from 0.3 to 0.6 $\mathrm{km} \mathrm{s}^{-1}$. Thus, roughly $1 / 2$ to $1 / 3$ of the observed line width can be attributed to thermal motion, with the remaining line width is due to bulk or non-thermal motions.

The integrated intensities and velocities (moment 1 ) of this region as observed with the VLBA with a resolution of $12.5 \times 6.3$ mas $\left(P . A .=-5^{\circ}\right)$ are shown in Figures $3 \mathrm{a}, \mathrm{b}$, c, and d. These results are in excellent agreement with the MERLIN results - W51_1 remains a single, though elongated maser feature with little velocity structure while W51_2 is composed of two distinct maser spots W51_2a, and W51_2b with velocities differing by only $\sim 0.09 \mathrm{~km} \mathrm{~s}^{-1}$ but with considerable velocity structure. The fitted line parameters from the VLBA data are given in Table 2, The fitted FWHM line width for W51_1 is in excellent agreement with that observed with MERLIN. The combined line width of the two components of W51_2 resolved by the VLBA is consistent with the MERLIN line width of this maser, although the individual VLBA line widths of W51_2a and W51_2b are slightly narrower.

We also fitted 2-D Gaussian components to the MERLIN and VLBA integrated intensity images in order to measure the maser locations, angular sizes, and flux densities. The results are given in Table 2. The masers were unresolved by the VLA and so the peak intensity $S d v($ peak) is equivalent to the integrated flux density $S d v(i n t)$. Interestingly, only about half of the VLA A-configuration integrated flux density is recovered by the MERLIN observations, while the VLBA and MERLIN recover a similar integrated flux density. If these masers are non-time variable, this suggests that MERLIN and the VLBA resolved out about half the total flux density. This suggests that these masers have a "core-halo" morphology as seen previously for $\mathrm{OH}(1720 \mathrm{MHz}), \mathrm{CH}_{3} \mathrm{OH}$, and $\mathrm{H}_{2} \mathrm{O}$ masers (see for example Hewitt et al. 2008; Minier et al. 2002; Richards et al. 2011). From the VLBA integrated intensity images, the devonvolved fitted sizes of the maser spots are W51_1: $37.3 \times 6.5$ mas (P.A.112 $2^{\circ}$, W51_2a: $13.7 \times 5.2$ mas (P.A.147 ${ }^{\circ}$ ), and W51_2b: $11.9 \times 4.2$ mas (P.A.127 $)$; with statistical uncertainties of about $10 \%$. For reference, at $6 \mathrm{kpc}, 10$ mas $\sim 10^{15} \mathrm{~cm}$ or $\sim 60$ AU. Using the peak intensities given in Table 2 these sizes correspond to peak brightness temperatures of $3.6 \times 10^{8}, 6.0 \times 10^{9}$, and $5.8 \times 10^{9} \mathrm{~K}$ for W51_1, W51_2a, and W51_2b, respectively. 


\subsubsection{Maser Polarization and Magnetic Field Properties}

From the MERLIN full Stokes polarization data, the maser spot W51_1 has a polarized intensity $\left(p=\operatorname{sqrt}\left(Q^{2}+U^{2}\right) / I\right)$ of $4.2 \pm 0.4 \%$ and a position angle of $\chi^{\text {maser }}=-1$. 6 , while the polarized intensity of W51_2 is $p=1.8 \pm 0.3 \%$ at $\chi^{\text {maser }}=4.4$. The uncertainty in the position angles $\left(\chi^{\text {maser }}\right)$ are dominated by the position angle calibration uncertainty which we estimate to be about $5^{\circ}$. The MERLIN linear polarization position angles are shown in Fig. 2a. Brogan et al. (2000) reported a polarized intensity of $3.5 \%$ and $\chi^{\text {maser }}(\mathrm{VLA})=-25^{\circ}$ for W51_2, but the polarization position angle calibration available for those data were very uncertain.

Following Brogan et al. (2000), we fit the Zeeman magnetic field strength using the thermal Zeeman equation $V=0.5 Z B_{\theta} d I / d \nu$ where $V$ is Stokes $V(\nu), Z=0.6536 \mathrm{~Hz}_{\mu} \mathrm{G}^{-1}$, and $d I / d \nu$ is the derivative of Stokes $I(\nu)$. The parameter $B_{\theta}=C|\vec{B}|$, is the magnitude of the magnetic field strength times a constant $C$ that may depend on the angle $\theta$ between the magnetic field $\vec{B}$ and the line-of-sight. For example, for thermal lines $C=\cos (\theta)$; the meaning of $C$ for the $\mathrm{OH}(1720 \mathrm{MHz})$ maser case will be discussed further in $\S 4.3$.

Figures 4 and 5 show the Zeeman fits for the MERLIN and VLBA data, respectively. The fitted values of $B_{\theta}$ are also listed in Table 2. The signal-to-noise of these fits are outstanding with $\mathrm{S} / \mathrm{N}$ up to 30 (MERLIN for W51_1) and S/N=7 (VLBA for W51_1). The observed values of $B_{\theta}$ from 1.5 to $2.2 \mathrm{mG}$ are in good agreement with the VLA observations

of Brogan et al. (2000). For the maser with the simplest velocity structure, W51_1, the MERLIN and VLBA magnetic field results are in excellent agreement. For W51_2, which the VLBA data reveal is composed of two spots, the MERLIN value is essentially an intensity weighted average of the two individual VLBA results.

These data are of sufficient quality that by following Hoffman et al. (2005a, b. b) we also independently derived $B_{\theta}$ by fitting Gaussian components to the left and right circularly polarized profiles independently and determining the line splitting directly, i.e. $B_{\theta}=\left(\nu_{R C P}-\right.$ $\left.\nu_{L C P}\right) / Z$. For example, for W51_2a the line splitting is $0.18 \pm 0.01 \mathrm{~km} \mathrm{~s}^{-1}$. This method yielded consistent results with the thermal Zeeman equation values listed in Table 2.

\subsection{VLA Radio Continuum}

The $90 \mathrm{~cm}(330 \mathrm{MHz})$ images presented in this work (for example Fig 19) are qualitatively similar to the $2^{\prime}$ resolution $90 \mathrm{~cm}$ image presented by Subrahmanyan \& Goss (1995), but the resolution and sensitivity are significantly improved. Figure 1 a shows the VLA $\mathrm{B}+\mathrm{C}+\mathrm{D}$ configuration $90 \mathrm{~cm}$ image of the W51 region (resolution $\sim 34^{\prime \prime}$, see Table 1 ) with 


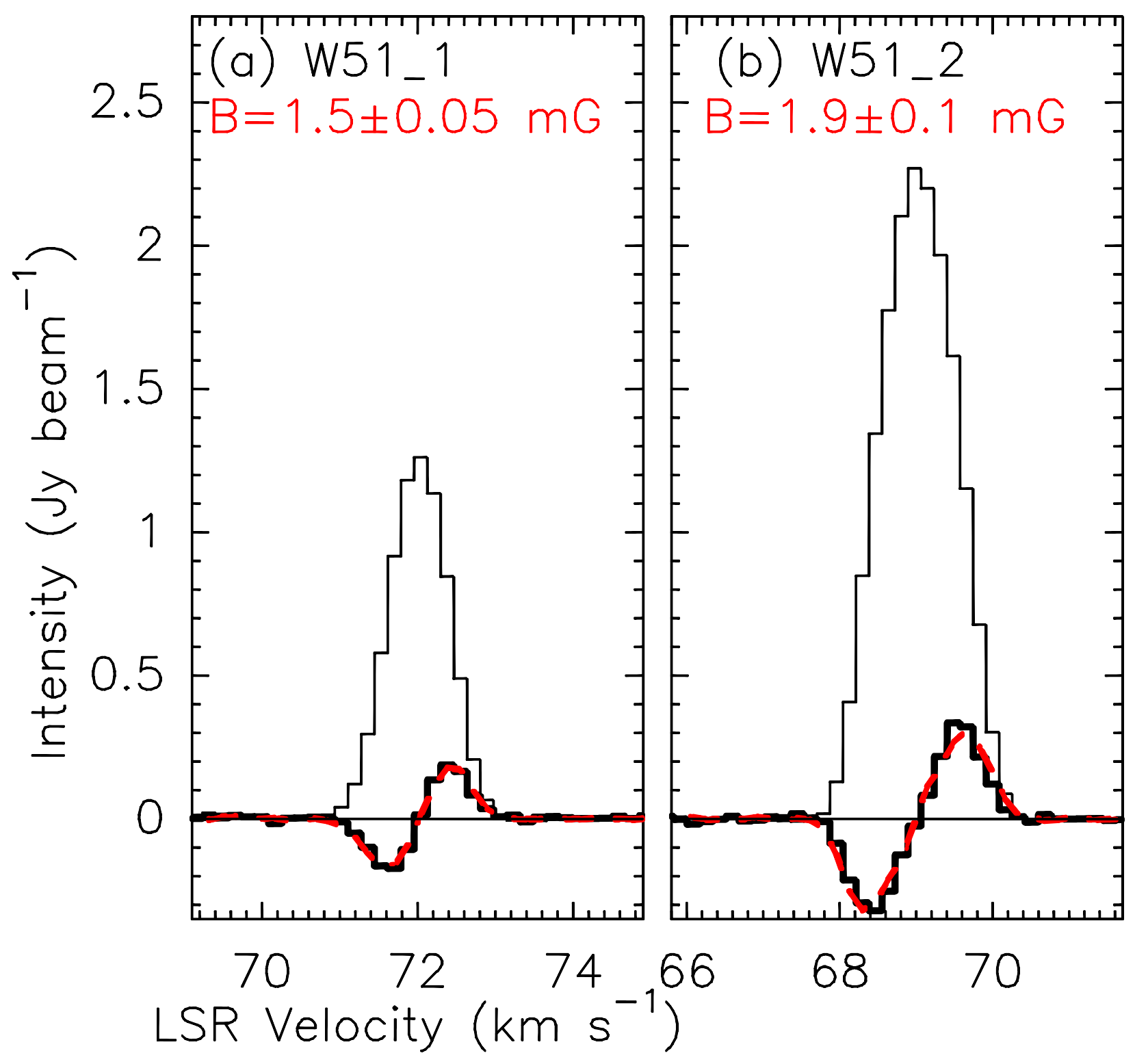

Fig. 4. - Single pixel profiles of the MERLIN Stokes I (thin solid, top), Stokes V (thick solid, bottom) and scaled derivative of Stokes I (dashed) at the peak positions of the (a) W51_1 and (b) W51_2 masers. The fitted magnetic field strengths and uncertainties are listed. 


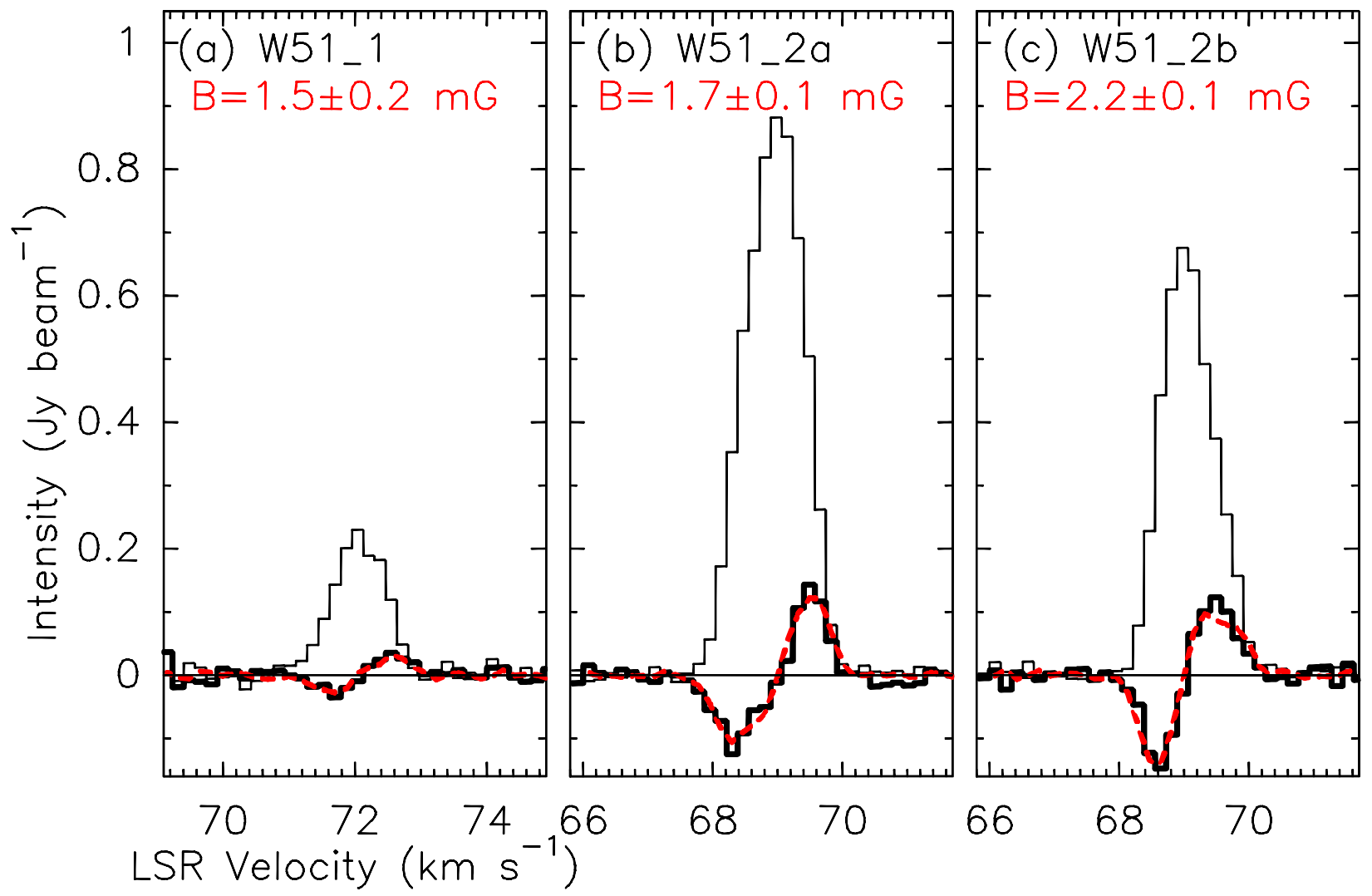

Fig. 5. - Single pixel profiles of the VLBA Stokes I (thin solid, top), Stokes V (thick solid, bottom) and scaled derivative of Stokes I (dashed) at the peak positions of the (a) W51_1, (b) W51_2a, and (c)W51_2b masers. The fitted magnetic field strengths and uncertainties are listed. 


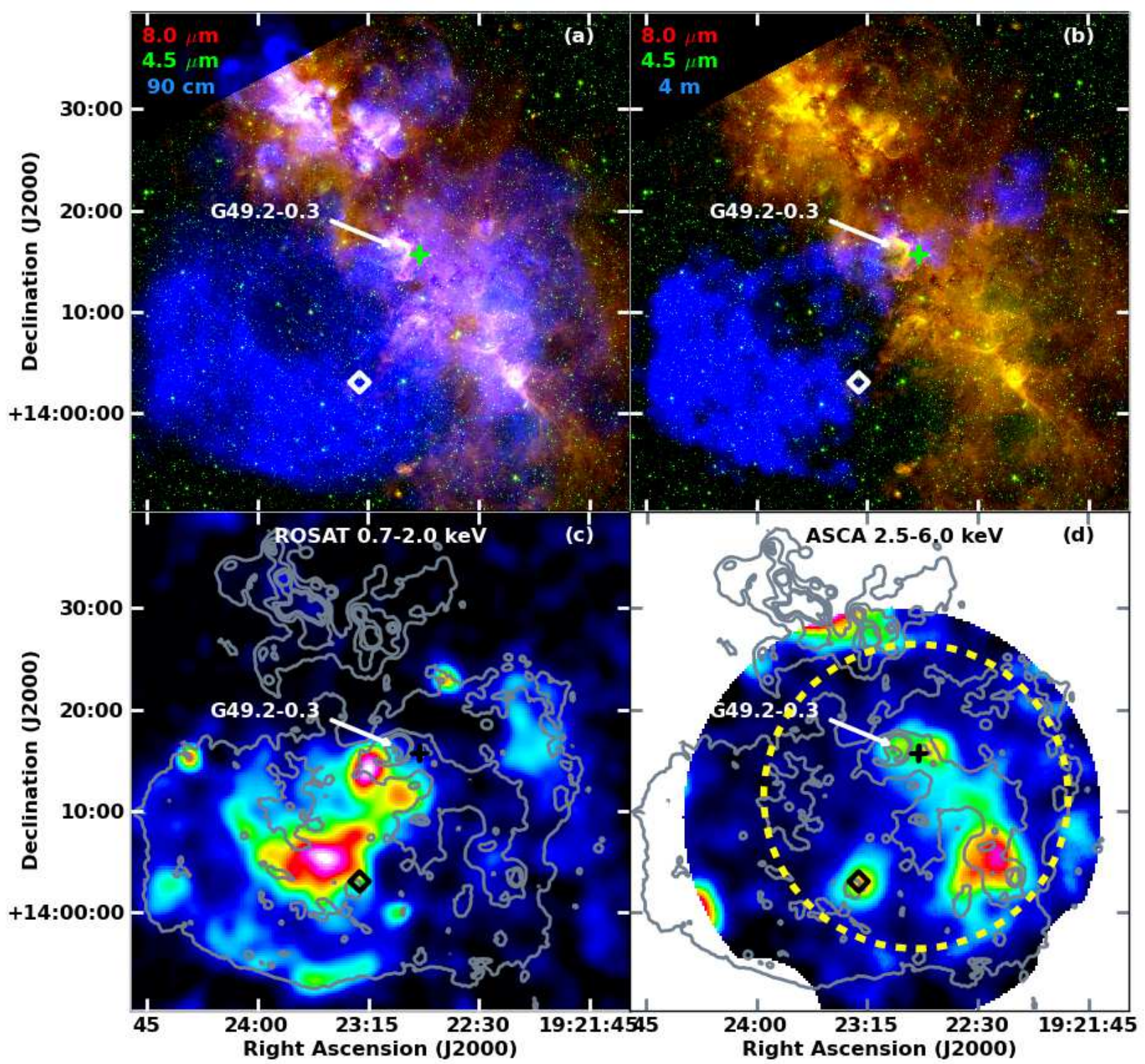

Fig. 6.- (a)-(b) Three color images of the W51 complex with red and green mapped to Spitzer 8.0 and $4.6 \mu \mathrm{m}$ from the GLIMPSE survey, respectively. In (a), blue is mapped to VLA B $+\mathrm{C}+\mathrm{D}$ configuration $90 \mathrm{~cm}$ data while in (b) blue is mapped to VLA B+C configuration $400 \mathrm{~cm}$ data. (c) ROSAT soft X-ray emission from 0.7 to $2.5 \mathrm{keV}$ (similar data are shown in Koo et al. (2002)). (d) $A S C A$ hard X-ray emission from 2.5 to $6.0 \mathrm{keV}$. The $A S C A$ data are from Koo et al. (2002). In (c) and (d), the grey contours are from the $90 \mathrm{~cm}$ image in (a) with levels of $60,150,300$, and $400 \mathrm{mJy} \mathrm{beam}^{-1}$. The $\mathrm{OH}(1720 \mathrm{MHz})$ maser locations are marked by a single green or black + symbol and the PWN candidate CXO J192318.5+1403035 from Koo et al. (2005) is marked by a black or white diamond. The location of the W51B H II region G49.2-0.3 is also indicated. Additionally, the region within which strong $\mathrm{TeV}$ emission was detected by MAGIC is indicated by the dashed yellow circle (Aleksić et al. 2012). 
the three major components W51A (H II region complex), W51B (H II region complex), and W51C (SNR) identified. The OH (1720 MHz) masers lie about 2'.3 west of the W51B H II region G49.2-0.3, and are coincident with an unresolved $90 \mathrm{~cm}$ source with an angular size of $\sim 1^{\prime}$ at a position of (J2000) 19h22m52.0s, +14 $15^{\prime} 50^{\prime \prime}$ (see Fig. 1a).

The W51 region is shown for the first time at a wavelength longer than $2 \mathrm{~m}$ in Figure 1b. The VLA $400 \mathrm{~cm} \mathrm{~B}+\mathrm{C}$ configuration image with a resolution of $\sim 88^{\prime \prime}$ (see Table 1) reveals diffuse, extended non-thermal emission concentrated mostly to the eastern side of the W51C SNR, with some diffuse emission also appearing toward the northwestern boundary of the W51C SNR. Most interesting for the purpose of the current study is the non-thermal $400 \mathrm{~cm}$ emission that appears to partially encircle the G49.2-0.2 H II region and the discovery of an unresolved $400 \mathrm{~cm}$ source coincident with $90 \mathrm{~cm}$ emission and the $\mathrm{OH}(1720 \mathrm{MHz})$ masers. Hereafter, we will call this source of non-thermal emission W51B_NT. It is notable that the area toward the southern portion of the W51B string of $\mathrm{H}$ II regions is invisible at $400 \mathrm{~cm}$, consistent with these regions being in the foreground of the W51C SNR, and free-free absorbing the $400 \mathrm{~cm}$ emission from W51C along this line-of-sight (see for example Brogan et al. 2005; Nord et al. 2006).

To aid comparison of the thermal vs non-thermal gas, Figures $6 \mathrm{a}$, b, shows three-color images constructed from the Spitzer GLIMPSE 4.5 and $8 \mu \mathrm{m}$ bands and the VLA $90 \mathrm{~cm}$ and $400 \mathrm{~cm}$ data, respectively. The mid-infrared emission traces thermal ionized gas, as well as emission from dust and PAHs (Benjamin et al. 2005). Its morphology matches closely that of the molecular cloud associated with W51B (see for example Koo 1999; Bieging et al. 2010). The $90 \mathrm{~cm}$ emission (Fig. 6a) traces both bright synchrotron (SNR) emission and optically thick free-free emission from H II regions. In contrast, the $400 \mathrm{~cm}$ emission (Fig. 6b) traces only synchrotron emission except where it is absorbed by foreground free-free emission. Fig. 6re and d show the large scale ROSAT soft and $A S C A$ hard X-ray emission, respectively, first presented by Koo et al. (2002). Soft X-rays trace hot $(\sim 0.3 \mathrm{keV})$ thermal gas from the SNR, except where it is absorbed by the intervening total column of atomic and molecular gas. In contrast, the hard X-rays are mostly unaffected by the column of gas along the line of sight and originates from even hotter thermal gas, including a pulsar wind nebula candidate and young stars with fast winds. We detect diffuse $90 \mathrm{~cm}$ emission at the location of the pulsar wind nebula candidate CXO J192318.5+1403035 from Koo et al. (2005, the location is indicated on Figs. 6a-d), but it is not clear if it arises from a distinct source compared to the overall diffuse SNR emission. Similar to the $400 \mathrm{~cm}$ emission, there is a lack of strong soft X-ray emission toward the southern portions of the W51B H II region complex. In Fig. 6d we also indicate the region in which strong $\mathrm{TeV}$ emission was detected by the MAGIC telescope (Aleksić et al. 2012). 


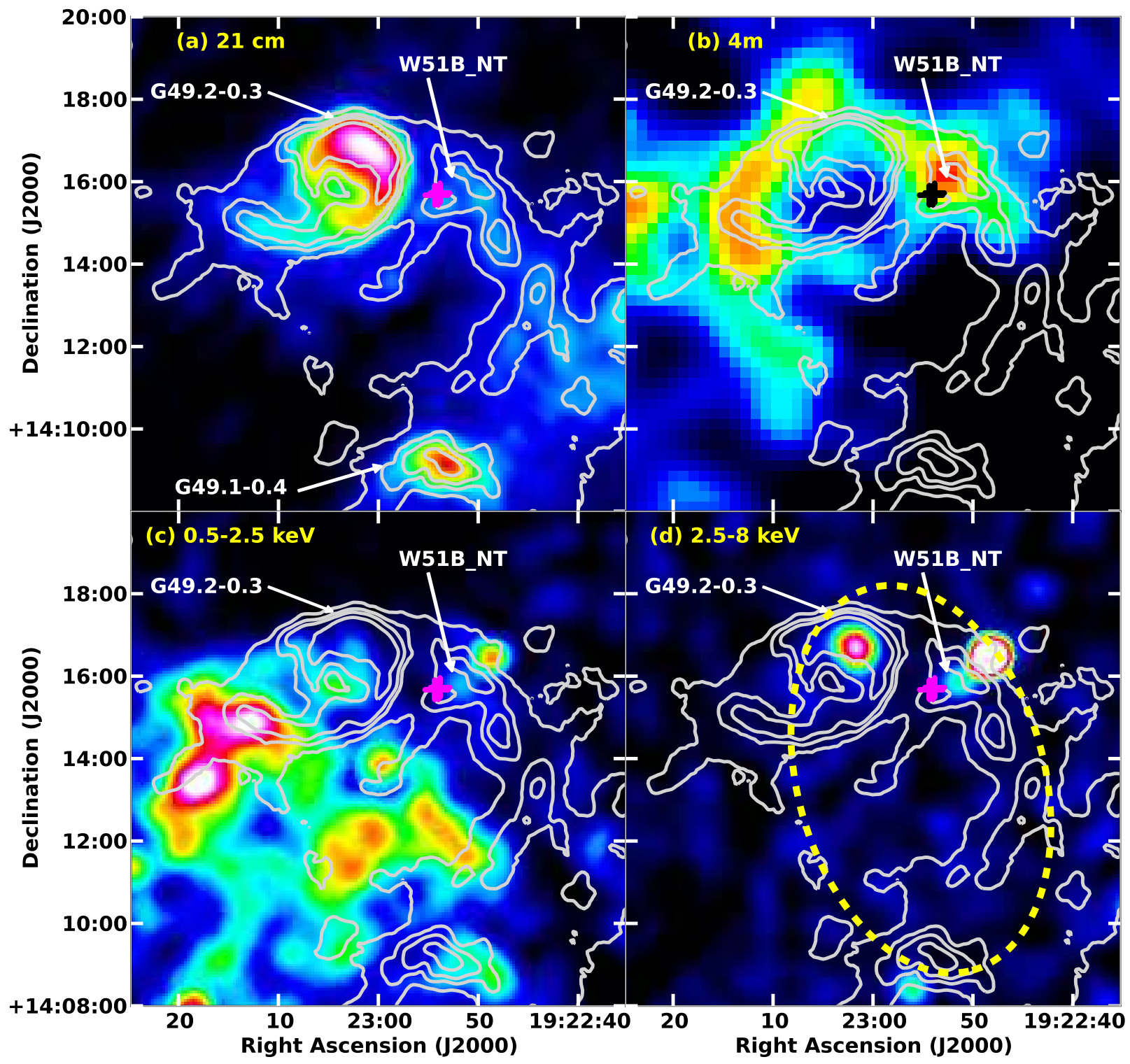

Fig. 7.- (a) VLA $21 \mathrm{~cm} \mathrm{C+D} \mathrm{configuration} \mathrm{continuum} \mathrm{image}\left(\sim 34^{\prime \prime}\right)$ using a log scale between -0.01 to $1.4 \mathrm{Jy}^{\text {beam }}{ }^{-1}$. (b) VLA $400 \mathrm{~cm} \mathrm{~B}+\mathrm{C}$ configuration image $\left(\sim 90^{\prime \prime}\right)$ using a linear scale between 0.15 and $1 \mathrm{Jy}$ beam $^{-1}$. (c) Chandra $0.5-2.5 \mathrm{keV}$ image. (d) Chandra 2.5-8.0 keV image. For both (c) and (d) a linear scale between $1.7 \times 10^{-8}$ to $1.4 \times 10^{-7}$ counts/s/pixel was used. All four panels are superposed with VLA 90cm A+B+C+D configuration contours at $33,55,77$, and $121 \mathrm{mJy} \mathrm{beam}^{-1}$. The $\mathrm{OH}(1720 \mathrm{MHz})$ maser locations are marked with magenta or black + symbols. The H II region G49.2-0.2 and the nonthermal radio source W51B_NT are also labeled on each panel; the H II region G49.1-0.4 is also labeled on (a). On (d) we also show a "test statistic" contour level of approximately 6 from the MAGIC 100 - $1000 \mathrm{GeV}$ emission (see Fig. 4 from Aleksić et al. 2012). 


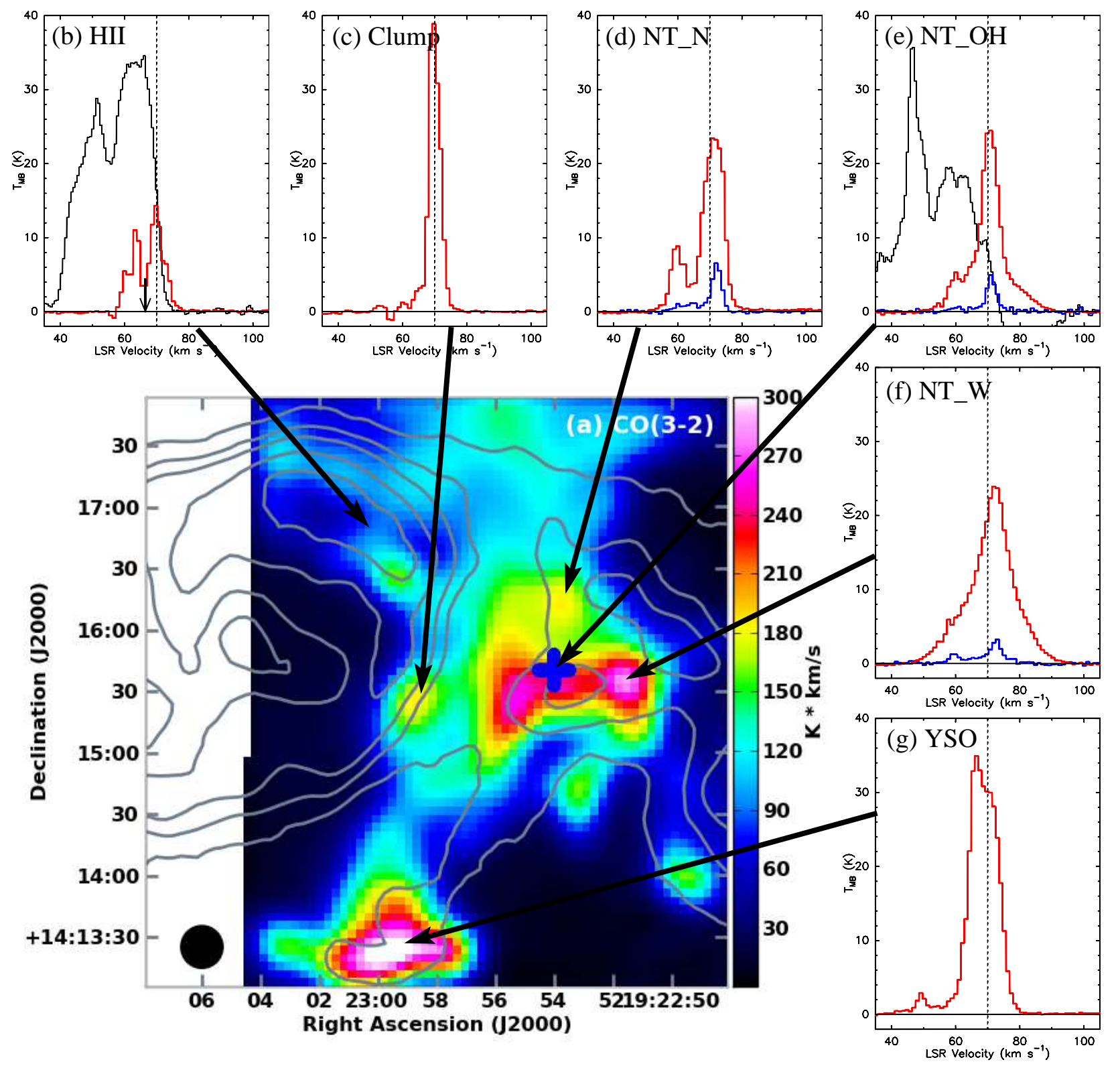

Fig. 8.- (a) Color image shows the $20^{\prime \prime}$ resolution integrated intensity of the JCMT CO(32) emission (velocity range 47 to $97 \mathrm{~km} \mathrm{~s}^{-1}$ ) with black VLA A+B+C+D $90 \mathrm{~cm}$ continuum contours superposed. The $90 \mathrm{~cm}$ contour levels are 33, 55, 77, 121, and $165 \mathrm{mJy} \mathrm{beam}^{-1}$. The location of the $\mathrm{OH}(1720 \mathrm{MHz})$ masers is indicated by a blue + symbol. The angular resolution of both images is $20^{\prime \prime}$ (beam is shown in lower left). (b)-(g) CO(3-2) (red), and where observed ${ }^{13} \mathrm{CO}(2-1)$ (blue), spectral line profiles taken from the indicated J2000 positions. Molecular emission appears to encircle W51B_NT and a dramatic increase in the $\mathrm{CO}(3-2)$ line width is evident in this region. The arrow at $67.2 \mathrm{~km} \mathrm{~s}^{-1}$ on the "H II" spectrum indicates its radio recombination line velocity from Lockman (1989). C-configuration VLA H I absorption line profiles are also shown (black) on the "H II" and "NT_OH" spectra (they have been multiplied by -100 , and -1000 , respectively). 
A zoomed in view of the $21 \mathrm{~cm}$ continuum emission in the vicinity of G42.9-0.3 and W51B_NT is shown in Figure $7 \mathrm{~h}$ at $\sim 34^{\prime \prime}$ resolution. For comparison, the $400 \mathrm{~cm}$ emission and archival soft and hard X-ray emission from Chandra (see Koo et al. 2005) of the same field are shown in Figures $7 \mathrm{~b}$, c, and d. These images show the coincidence of 21, 90, and $400 \mathrm{~cm}$ emission toward W51B_NT, as well as the presence of a faint hard X-ray source at this position. Unfortunately, this emission is too weak to extract a useful spectrum. The nature of the stronger hard X-ray source to the NW of W51B_NT is unknown. After accounting for the background contribution we estimate integrated flux densities for W51B_NT (defined by the $90 \mathrm{~cm} 0.15 \mathrm{Jy}_{\text {beam }}{ }^{-1}$ contour shown in Fig. 6a, though it is partially obscured by the maser symbol) of $\sim 0.5,1.3$, and $0.6 \mathrm{Jy}$ at $400 \mathrm{~cm}, 90 \mathrm{~cm}$, and $21 \mathrm{~cm}$, respectively. The uncertainty of these flux densities are dominated by the background estimate, and are not accurate to better than $\pm 0.2 \mathrm{Jy}$. The low $400 \mathrm{~cm}$ flux density is almost certainly a result of free-free absorption along this complex line-of-sight (see for example Brogan et al. 2005). The 21 and $90 \mathrm{~cm}$ flux densities imply a non-thermal radio spectral index of $-0.7 \pm 0.4$ $\left(S_{\nu} \propto \nu^{\alpha}\right)$.

Other groups have searched for possible non-thermal sources in the W51 complex. In particular, Moon \& Koo (1994) used the Bonn $11 \mathrm{~cm}$ Galactic plane survey (resolution 4.2') along with IRAS $60 \mu \mathrm{m}$ images to disentangle the thermal and non-thermal components of W51. Though uncertain with respect to absolute flux densities, their resulting images show the well-known thermal sources in W51A and W51B as well as the non-thermal W51C SNR. Interestingly, they also see evidence for a non-thermal source west of the G49.2-0.3 H II region. More qualitative evidence for the existence of non-thermal emission in the vicinity of W51B_NT was presented by Copetti \& Schmidt (1991) at $151 \mathrm{MHz}$ with a resolution of $2^{\prime} \times 5^{\prime}$ with a double source detected near G49.2-0.3 with a separation of a few arcminutes (note that the published declination scale of the $151 \mathrm{MHz}$ image must be displaced to the south by 5 arc min Subrahmanyan \& Goss 1995). Though suggestive, these early data have too poor an angular resolution to be useful in the current analysis.

\subsection{Thermal Molecular Gas Properties}

The JCMT CO(3-2) integrated intensity from 40 to $95 \mathrm{~km} \mathrm{~s}^{-1}$ with a resolution of $20^{\prime \prime}$ is shown in Figure $8 \mathrm{a}$. Representative spectra are shown in Figs. 8 b-g), and where observed, the ${ }^{13} \mathrm{CO}(2-1)$ spectra are also shown (Figs. 8 $8 \mathrm{~d}-\mathrm{f}$ ). From these data we find that $\mathrm{CO}$ emission partially encircles W51B_NT with the strongest emission along the eastern and southern boundaries. This enhanced $\mathrm{CO}(3-2)$ emission is coincident with the locations of the $\mathrm{OH}(1720 \mathrm{MHz})$ masers which are located towards the SE corner of the W51B_NT 
source (Fig. 8b, profile "NT_OH" is taken from the maser location). The spectra in the W51B_NT region (Figs. 8d, e, f) show at least three components: (I) a narrow $(\Delta v \sim 5$ $\left.\mathrm{km} \mathrm{s}^{-1}\right)$, but strong feature $T_{M B} \sim 20 \mathrm{~K}$ at $\sim 71 \mathrm{~km} \mathrm{~s}^{-1} ;$ (II) a broad $\left(\Delta v \sim 20 \mathrm{~km} \mathrm{~s}^{-1}\right.$ wide) feature with a strength of $T_{M B} \sim 10 \mathrm{~K}$ also centered around $70 \mathrm{~km} \mathrm{~s}^{-1}$; and (III) a narrow $\left(\Delta v \sim 4 \mathrm{~km} \mathrm{~s}^{-1}\right)$, weak blue-shifted feature at $60 \mathrm{~km} \mathrm{~s}^{-1}$. The appearance of the dramatically broadened component (II) in the vicinity of W51B_NT is strongly indicative of a shock. The $\mathrm{CO}$ throughout the mapped region has an average peak velocity of about $70 \mathrm{~km} \mathrm{~s}^{-1}\left( \pm 3 \mathrm{~km} \mathrm{~s}^{-1}\right)$, in agreement with components (I) and (II) toward the masers. Thus, while the broad velocity width observed toward the W51B_NT region is exceptional, it does not appear that the W51B_NT region arises from a distinct cloud along the line of site, but is co-distant with the nearby $\mathrm{H}$ II regions like G49.2-0.3. Additionally, the velocities of the narrow and broad $\mathrm{CO}$ components at 70 and $71 \mathrm{~km} \mathrm{~s}^{-1}$, respectively, are in excellent agreement with the range of $\mathrm{OH}(1720 \mathrm{MHz})$ maser velocities (69-72 $\mathrm{km} \mathrm{s}^{-1}$; see Table 2).

Other features of note in the full $\mathrm{CO}(3-2)$ raster (see Fig. $8 \mathrm{~b}-\mathrm{g}$ ): (i) the emission associated with the G49.2-0.3 H II region is comparatively weak and is composed of two main components that bracket the radio recombination line velocity from Lockman (1989) of 67.2 $\mathrm{km} \mathrm{s}^{-1}$. (ii) Along the SW rim of the H II region is a compact "clump" with the strongest $\mathrm{CO}(3-2)$ emission in the mapped region $(40 \mathrm{~K})$, but with narrow $\left(4 \mathrm{~km} \mathrm{~s}^{-1}\right)$ line widths. (iii) The southernmost $\mathrm{CO}(3-2)$ clump labelled "YSO" shows evidence of bipolar outflow emission predominantly in an east-west direction, but with a third component to the north. The "YSO" source is also associated with weak $90 \mathrm{~cm}$ and $20 \mathrm{~cm}$ emission, though its appearance is unremarkable in the mid-IR. From these clues, it seems likely this source is an intermediate to massive protostar with the radio continuum arising from optically thick free-free emission.

The appearance of the $\mathrm{CO}(3-2)$ and ${ }^{13} \mathrm{CO}(2-1)$ spectra toward W51B_NT, with a broad $\left(\Delta v \sim 20 \mathrm{~km} \mathrm{~s}^{-1}\right)$ component superposed on a narrow $\left(\Delta v \sim 5 \mathrm{~km} \mathrm{~s}^{-1}\right)$ feature at the LSR velocity is in good agreement with other $\mathrm{CO}(3-2)$ studies of SNR OH $(1720 \mathrm{MHz})$ maser regions (see for example Frail \& Mitchell 1998; Reach et al. 2002). To facilitate comparison of all four observed thermal molecular line species, we convolved the $\operatorname{JCMT~} \mathrm{CO}(3-2)$ and ${ }^{13} \mathrm{CO}(2-1)$ cubes to 26 " resolution to match the $\mathrm{CSO} \mathrm{HCN}(3-2)$ and $\mathrm{HCO}^{+}(3-2)$ data. Spectra of all four species at this resolution toward the centroid of the $\mathrm{OH}$ maser location are shown in Figure 9a, and the parameters from Gaussian fits using the CASA task specfit are given in Table 3 . 


\section{DISCUSSION}

\subsection{Physical Conditions in the Pre- and Post-shock Gas}

Using the $26^{\prime \prime}$ resolution fitted parameters at the $\mathrm{OH}$ maser position (Table 3), we ran the RADEX radiative transfer code (van der Tak et al. 2007) with the LAMDA molecular data files (Schöier et al. 2005) to simultaneously model the $\mathrm{CO}(3-2)$ and ${ }^{13} \mathrm{CO}(2-1)$ line strengths and thereby estimate the physical parameters of the pre-shock and post-shock gas. Because these two lines trace somewhat different excitation conditions, combining observations of both lines helps to constrain the possible densities and temperatures present. We chose the slab option in RADEX as it is the most appropriate geometry for both a shock and a molecular cloud filament. We ran grids of RADEX models over density and temperature for a range of plausible column densities, while assuming $\mathrm{N}(\mathrm{CO}) / \mathrm{N}\left(\mathrm{H}_{2}\right)=10^{-4}$ and an isotopic abundance of ${ }^{12} \mathrm{C} /{ }^{13} \mathrm{C}=60$ (Milam et al. 2005). The $\mathrm{H}_{2}$ column densities and corresponding densities and kinetic temperatures that simultaneously produce $\mathrm{CO}(3-2)$ and ${ }^{13} \mathrm{CO}(2-1)$ antenna temperatures that match the observed spectra are plotted in Fig.9b. Next we used RADEX to determine what abundance of $\mathrm{HCN}(3-2)$ and $\mathrm{HCO}^{+}(3-2)$ would be required to produce the observed intensities of these high density tracers at the densities and kinetic temperatures derived from the $\mathrm{CO}$ observations.

Figure 9b summarizes the results for the four species modeled. In the lower left corner of Fig. 9b, the sequence of points of varying $\mathrm{H}_{2}$ column density (from $4 \times 10^{21}$ to $6 \times 10^{21} \mathrm{~cm}^{-2}$ ) indicate the conditions that reproduce the observed line profiles in the pre-shock gas, with kinetic temperatures ranging from 26 to $32 \mathrm{~K}$ and $\mathrm{H}_{2}$ densities of 850 to $4000 \mathrm{~cm}^{-3}$. The corresponding $\mathrm{HCO}^{+}$and $\mathrm{HCN}$ line strengths are consistent with abundances of $1-2 \times 10^{-8}$, which are typical of the values seen in massive star formation regions (Doty et al. 2002, and references therein).

We can compare the pre-shock column densities derived from the RADEX analysis with those estimated from previous millimeter continuum and X-ray observations toward this region. W51B_NT is not detected at $1.1 \mathrm{~mm}$ in the $33^{\prime \prime}$ resolution Bolocam Galactic Plane Survey (the H II region G49.2-0.3 is detected; see Aguirre et al. 2011, for Survey details). The $5 \sigma$ upper limit measured in the vicinity of W51B_NT is about $0.36 \mathrm{mJy}_{\text {beam }}^{-1}$ after multiplying by the 1.5 flux density correction factor recommended by Aguirre et al. (2011). Assuming the dust temperature is about $T_{\text {dust }}=25 \mathrm{~K}$ in the pre-shock gas, a dust to gas ratio of 100, and a dust opacity of $0.0114 \mathrm{~cm}^{2} \mathrm{~g}^{-1}$ (Ossenkopf \& Henning 1994; Aguirre et al. 2011), we find a dust-derived $N\left(\mathrm{H}_{2}\right)$ column density upper limit of $\sim 8 \times 10^{21} \mathrm{~cm}^{-2}$. We can also use the results of previous X-ray observations toward this region to derive a lower

limit. Using the Suzaku X-ray satellite, Hanabata et al. (2012) find an average total proton 
column density $N_{p}=N(\mathrm{HI})+2 N\left(\mathrm{H}_{2}\right)$ toward their Region 3, which encompasses most of W51B including W51B_NT, of $2.4 \times 10^{22} \mathrm{~cm}^{-2}$. Using a combination of Arecibo and VLA H I emission and absorption data, Koo (1997) find $N(H I) \sim 1.9\left(T_{\text {spin }} / 160 \mathrm{~K}\right) \times 10^{22} \mathrm{~cm}^{-2}$ along the line-of-sight to the nearby H II region G49.2-0.3, with $80 \%$ of the total column originating close to the W51B region. Since $T_{\text {spin }} \sim 160 \mathrm{~K}$ is an upper limit, $1.9 \times 10^{22}$ represents an upper limit to the true $\mathrm{N}(\mathrm{HI})$. When compared to the X-ray derived $N_{p}$, this suggests $N\left(\mathrm{H}_{2}\right) \geq 2.5 \times 10^{21} \mathrm{~cm}^{-2}$. Thus, the allowed range estimated from previous observations is $(2.5-8) \times 10^{21} \mathrm{~cm}^{-2}$, compared to $N\left(\mathrm{H}_{2}\right)=(4-6) \times 10^{21} \mathrm{~cm}^{-2}$ from the RADAX molecular line analysis.

The post-shock gas is represented in the upper portion of Figure 9b, with the gray shaded rectangular region indicating the theoretical range of parameters sufficient to pump the $1720 \mathrm{MHz} \mathrm{OH}$ maser. A beam filling factor of significantly less than 1 is required to explain the low observed line strengths in the broad $\mathrm{CO}$ component. This result is not surprising given that the $26^{\prime \prime}$ beam corresponds to a linear diameter of $2.3 \times 10^{18} \mathrm{~cm}$, because the width of the region of elevated temperature in a typical interstellar C-type shock is $\sim 10^{16}-10^{17} \mathrm{~cm}$, depending on the ionization rate (Wardle 1999). The $\mathrm{HCO}^{+}$abundance is significantly lower (factors of 3-20) throughout the shaded region than its value in the pre-shock gas, suggesting a difference in chemistry. Indeed, $\mathrm{PDR}$ models indicate that the $\mathrm{HCO}^{+} / \mathrm{CO}$ abundance ratio drops precipitously when the ionization rate exceeds $10^{-15} \mathrm{~s}^{-1}$ (Ceccarelli et al. 2011). As mentioned previously, the W51B/W51C interface region is a strong emitter of $\gamma$-rays with properties that are consistent with a hadronic emission mechanism (see Aleksić et al. 2012, and references therein). Hewitt et al. (2009) estimate $\gamma$-ray and X-ray induced ionization rates of $4.4 \times 10^{-16} \mathrm{~s}^{-1}$ and $8.8 \times 10^{-16} \mathrm{~s}^{-1}$, respectively, for this region. Though dependent on many assumptions, together, the $\gamma$-ray plus X-ray induced ionization rate is about 30 times the local interstellar rate, and is in excellent accord with both that needed to produce an adequate abundance of $\mathrm{OH}$ for the masers (Wardle 1999), and that required to lower the $\mathrm{HCO}^{+} / \mathrm{CO}$ abundance as predicted by Ceccarelli et al. (2011).

Similar to $\mathrm{HCO}^{+}$, the inferred abundance of $\mathrm{HCN}$ is also reduced in much of the postshock parameter space, with the exception of the low-temperature, low-density end of the range (see Fig. 9b). While this suggests that the physical conditions in the post-shock gas may lie in this triangular region, it may be that the abundance of HCN is also altered by the shock chemistry. In any case, a range of physical conditions almost certainly exists across the shock, such that there is no single unique answer. Still these observations demonstrate that the range of expected densities and temperatures from the maser models are supported by the thermal molecular line data. 

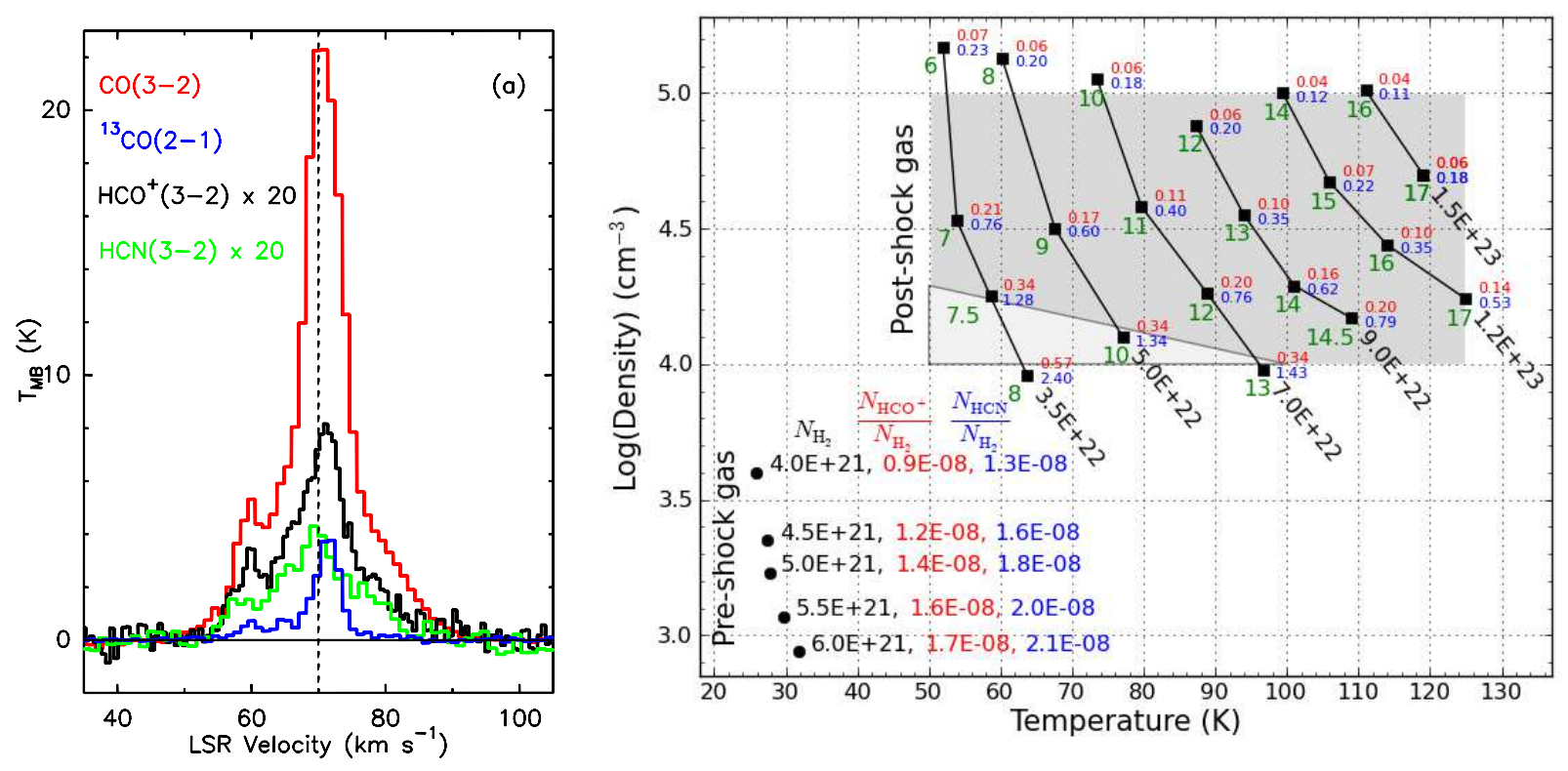

Fig. 9.- (a) Molecular line spectra with $26^{\prime \prime}$ resolution towards the maser position; the $\mathrm{HCO}^{+}$and $\mathrm{HCN}$ have been multiplied by 20 to improve their visibility. (b) Results of the RADEX radiative transfer models of the number density vs. temperature for the thermal gas toward the $\mathrm{OH}$ maser position based on the fitted $\mathrm{CO}$ and ${ }^{13} \mathrm{CO}$ line profiles. The gray shaded rectangular region indicates the theoretical range of parameters sufficient to pump the $1720 \mathrm{MHz} \mathrm{OH}$ maser. Filled circles denote the results for the narrow velocity component from the pre-shock gas and are labelled by the $\mathrm{H}_{2}$ column density (assuming a beam filling factor of 1.0), and the corresponding abundances of $\mathrm{HCO}^{+}$and $\mathrm{HCN}$. Filled squares denote the results for the broad velocity component from the post-shock gas. The lines connect the results for the same $\mathrm{H}_{2}$ column density and the numbers in green to the left of the squares indicate the inverse of the beam filling factor. The numbers in blue and red denote the inferred $\mathrm{HCN}$ and $\mathrm{HCO}^{+}$abundances, respectively, in units of $10^{-8}$. 


\subsection{Nature of W51B_NT}

First, to summarize the key multi-wavelength results: a non-thermal source of low frequency radio emission (W51B_NT) has been detected in the vicinity of the W51B OH (1720 $\mathrm{MHz}$ ) masers. This source is partially encircled by shocked molecular gas that is coincident in space and velocity with the masers. Moreover, detailed modeling of the molecular emission indicates that the physical conditions in the pre- and post-shock gas are in good agreement with that expected from collisional pump models. It is also notable that the molecular gas associated with W51B_NT shares a similar LSR velocity with nearby H II regions (for example G49.2-0.3), suggesting that the masers are co-distant with the northern members of the W51B H II region complex. The non-thermal radio source is also coincident with a hard X-ray source and falls within the region of high likelihood for the position of TeV $\gamma$-ray emission, both of which have been suggested as necessary to produce enough $\mathrm{OH}$ abundance (from water) in C-type shocks for SNR OH (1720 MHz) maser emission. Thus, the evidence indicates that these $\mathrm{OH}(1720 \mathrm{MHz})$ masers are of the $\mathrm{SNR} /$ molecular cloud interaction variety.

The multi-wavelength data presented in this work suggests two possible scenarios for the nature of W51B_NT: (1) the non-thermal radio continuum emission, CO shock, $\mathrm{OH}$ $(1720 \mathrm{MHz})$ masers, and hard X-ray emission are due to the W51C SNR, which lies close enough behind W51B for its shock wave to have hit the H II region complex; or (2) along this crowded, tangent point line of sight there is a previously unknown small diameter $\left(\sim 2^{\prime}\right)$ supernova remnant co-distant with the W51B H II regions. As will be described below, option (1) seems the most consistent with the full range of data available for this region.

It is clear from both $4 \mathrm{~m}$ and soft X-ray absorption that the W51B H II region complex lies at least partially in front of the W51C SNR. The key uncertainty is whether W51B is close enough to W51C for the SNR's shock front to have reached its back side. In addition to the presence of W51B_NT and the $\mathrm{OH}(1720 \mathrm{MHz})$ masers, there is further evidence that the W51C SNR is interacting with much of the northern end of W51B. For example, Koo (1997) report a filamentary arc of high velocity (HV), shocked H I emission at velocities between $\sim 80-140 \mathrm{~km} \mathrm{~s}^{-1}$ toward the northern section of W51B. This arc of emission begins just west of W51B_NT, curves around to the south, and ends just north of the H II region G49.1-0.4. Figure 10a shows the morphology of the HV H I compared to the $90 \mathrm{~cm}$ continuum; the $\mathrm{CO}(3-2)$ emission from the smaller field of view imaged with the JCMT is also shown. The arc of the filament matches very closely with the morphology of the $90 \mathrm{~cm}$ continuum emission in this region. Koo \& Moon (1997) also find HV CO(1-0) emission roughly co-spatial with the $\mathrm{HV} \mathrm{H} \mathrm{I} \mathrm{(most} \mathrm{of} \mathrm{the} \mathrm{HV} \mathrm{CO}(1-0)$ is outside the field of view of the $\mathrm{CO}(3-2)$ JCMT data, see Fig. 10a). These authors suggest that the HV gas 


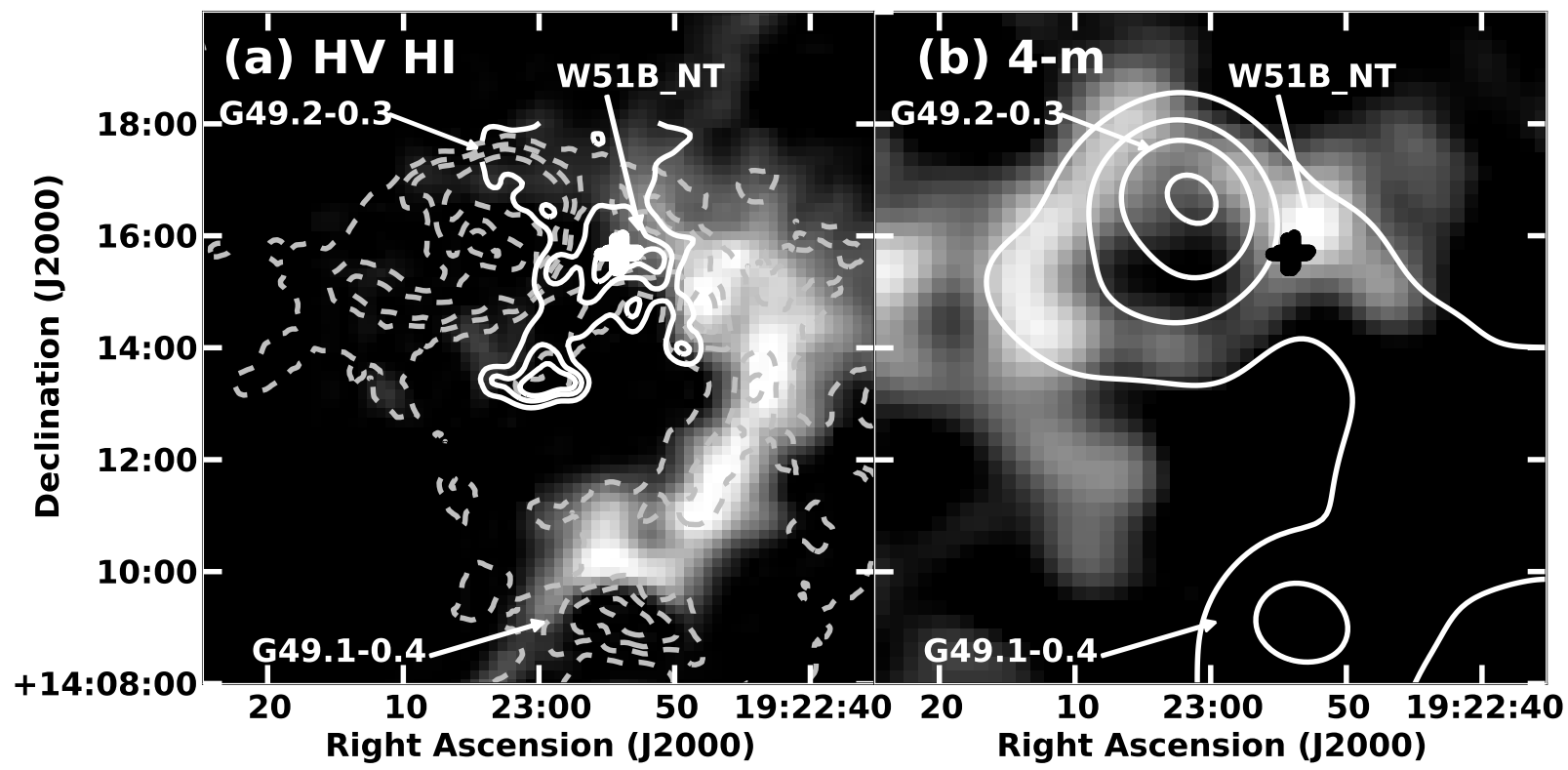

Fig. 10.- (a) Greyscale H I integrated intensity image from 82 to $140 \mathrm{~km} \mathrm{~s}^{-1}$ showing the high velocity (HV) shocked HI emission from Koo (1997). Superposed are dashed VLA $\mathrm{A}+\mathrm{B}+\mathrm{C}+\mathrm{D} 90 \mathrm{~cm}$ continuum contours at $11 \times(3,5,7,11$, and 15$) \mathrm{mJy}_{\text {beam }}{ }^{-1}$ and solid white JCMT CO(3-2) integrated intensity (from 47 to $97 \mathrm{~km} \mathrm{~s}^{-1}$ ) contours at 60, 140, and $220 \mathrm{~K}^{*} \mathrm{~km} \mathrm{~s}^{-1}$ over its smaller field of view, see Fig. 8. (b) Greyscale image of the $400 \mathrm{~cm}$ emission with a linear color scale from $0.3(3 \sigma)$ to $0.8 \mathrm{Jy}_{\text {beam }}{ }^{-1}$, with white $\tau_{4 m}$ contours at $1.5,5,10$, and 20 superposed. The coincidence of detectable $400 \mathrm{~cm}$ emission where the predicted $\tau_{4 m}$ is high suggests the H II region cannot be entirely in front of the W51C SNR. The locations of the $\mathrm{OH}(1720 \mathrm{MHz})$ masers are indicated by white (a) or black (b) + symbols. 
is tracing a shock interaction between the W51C SNR and the molecular gas associated with the (foreground) W51B complex of H II regions. Indeed, the HV H I does appear to lie along the transition between the unabsorbed $400 \mathrm{~cm}$ SNR emission and the large scale filamentary molecular cloud associated with the W51B complex - the CO filament is roughly coincident with the Spitzer $8 \mu \mathrm{m}$ emission shown in Fig. 6a, b (see for example Koo 1999; Bieging et al. 2010). Koo (1997) finds that the properties of the HV H I shock are consistent with a dissociative J-type shock, and further postulate that the post-shock gas is mostly atomic since the HV H I column density is quite high. Therefore, the non-detection of HV H I toward W51B_NT is not surprising since it is spatially coincident with the $\mathrm{OH}$ (1720 MHz) masers that are thought to arise in non-dissociative C-type shocks (Wardle 1999).

Another piece of evidence indicating a more extensive interaction is the morphology of the $400 \mathrm{~cm}$ emission. In particular, the non-thermal $400 \mathrm{~cm}$ emission appears to both encircle and partially overlap with the G49.2-0.3 H II region emission as defined by the shorter wavelength $21 \mathrm{~cm}$ image (see Figs,7a,b). As described, for example, in Brogan et al. (2005) and Nord et al. (2006), relatively little thermal ionized gas is required to absorb $400 \mathrm{~cm}$ emission so it would be surprising to detect such low frequency emission in the direction of a foreground H II region. This expectation can be quantified by comparing an estimate of the $400 \mathrm{~cm}$ free-free continuum opacity from the H II region with the observed $400 \mathrm{~cm}$ emission. The $400 \mathrm{~cm}$ opacity was calculated by first convolving the $\mathrm{C}+\mathrm{D} 21 \mathrm{~cm}$ continuum image to the $400 \mathrm{~cm}$ image resolution of $\sim 90^{\prime \prime}$, and then converting this image to brightness temperature. Next the $\tau_{21 \mathrm{~cm}}$ was calculated from $T_{b}=T_{e}(1-\exp (-\tau))$ assuming an electron temperature of $10,000 \mathrm{~K}$, and then extrapolated to $\tau_{4 m}$ using $\tau_{\lambda}=\tau_{21 \mathrm{~cm}}(\lambda(\mathrm{cm}) / 21 \mathrm{~cm})^{2.1}$. Using this method, we find a peak $400 \mathrm{~cm}$ opacity of 22.5 with $\tau_{4 m}>1$ extending well into the observed $400 \mathrm{~cm}$ emission, see Figure 10 b. Of course, in reality the $T_{e}$ within the $\mathrm{H}$ II region is unlikely to be constant, but the relatively high assumed value yields a lower limit to $\tau_{21 \mathrm{~cm}}$. The strongest observed $400 \mathrm{~cm}$ emission is on the eastern side of W51C at $1.35 \mathrm{Jy}$ beam $^{-1}$ (see Fig. 1b). Since it seems unlikely that the unabsorbed $400 \mathrm{~cm}$ emission underlying the $\mathrm{HII}$ region is significantly brighter than this intensity, we take it to be an upper limit to the unabsorbed $400 \mathrm{~cm}$ non-thermal emission toward G49.2-0.3. When this assumption is coupled with the $3 \sigma \mathrm{rms}$ noise of the $400 \mathrm{~cm}$ image of $0.3 \mathrm{Jy}^{\mathrm{beam}}{ }^{-1}$, we find that lines of sight with $\tau_{4 m}>1.5$ would be undetectable if the $H$ II region lies entirely in front of the $400 \mathrm{~cm}$ non-thermal emission. However, as demonstrated in Fig. 10b detectable $400 \mathrm{~cm}$ emission is observed toward regions with $\tau_{4 m}$ up to $\sim 20$ ! This analysis suggests, as does the morphology, that in fact the W51C SNR has partially enveloped the G49.2-0.3 H II region.

Interestingly, from analysis of the optical extinction toward the W51B region, Han (2001) found that the northern half of W51B is more heavily extincted than the south/southwestern 
end. Since the total molecular column density is actually larger to the SW, these authors (also see Koo 1999) suggest that the northern end of W51B is tilted deeper into the cloud than the south/southwestern end, placing it closer to W51C. From all of these comparisons it seems clear that the W51C SNR is interacting with the northern part of the W51B H II region complex.

\subsection{Constraints on Magnetic Field Direction}

As a shock propagates, the gas is compressed in the direction the shock is moving and the component of the magnetic field parallel to the shock front will be amplified. For collisionally pumped maser emission it is further expected that the shock must be moving more or less perpendicular to our line-of-sight in order to assure adequate velocity coherence, and column density along the line-of-sight. Thus, collisionally pumped masers like $\mathrm{OH}$ (1720 $\mathrm{MHz}$ ) masers associated with SNRs and Class $\mathrm{I} \mathrm{CH}_{3} \mathrm{OH}$ masers are always found near the systemic velocity. These two facts together imply that the magnetic field vector is oriented somewhere in the plane perpendicular to the direction the shock front is moving, and we are viewing that shock front edge-on. Using this as the starting geometry, one would like to use the polarization measurements to constrain the direction of the magnetic field vector and the value of $C$ in the $B_{\theta}=C \mid \vec{B}$ |equation.

Maser propagation theory (see e.g. Elitzur 1998, 1996; Watson \& Wyld 2001) suggests that the observed percentage of linear polarization $p$ is a strong function of the angle between the line-of-sight and the magnetic field vector $\theta_{\text {los }}$. Indeed, for $\theta_{\text {los }}=55^{\circ}$ the observed $p$ should be zero (it is also zero for $\theta_{l o s}=0^{\circ}$ ). Away from the critical angles of $55^{\circ}$ and $0^{\circ}, p$ is non-zero, though its magnitude is a strong function not only of $\theta_{\text {los }}$, but also the saturation level of the maser and to some extent to the angular momentum of the molecular state that is masing. The brightness temperatures of $\mathrm{OH}(1720 \mathrm{MHz})$ masers $T_{b} \sim 10^{8-10} \mathrm{~K}$, along with the lack of significant variability are consistent with at least a moderate degree of saturation (also see additional arguments in Brogan et al. 2000; Hoffman et al. 2005a,b). Assuming moderate saturation, according to the model of Watson \& Wyld (2001), for a $\mathrm{J}=2-1$ state, $p$ is expected to be within about $-10 \%$ to $+20 \%$, with positive values corresponding to $\theta_{\text {los }}>55^{\circ}$ and negative values corresponding to $\theta_{\text {los }}<55^{\circ}$. If $\theta_{\text {los }}$ is known, this model can also be used to constrain the $C$ parameter (which may depend on $\theta$ ) in the Stokes $\mathrm{V}$ conversion to magnetic field strength. For small values of $p$ and moderate saturation, $V \approx(Z / 2) B d I / d \nu(\mathrm{Z}=$ the Zeeman coefficient, and $B$ is the total field strength) with no $\theta$ dependence, i.e. $C \approx 1$ and hence $B \approx B_{\theta}$. Currently, no detailed analysis is available for the $\mathrm{J}=3 / 2 \mathrm{OH}(1720 \mathrm{MHz})$ transition, though it would likely be qualitatively similar to the 
$\mathrm{J}=2-1$ case (private communication, William Watson 2005). This suggests that given the small measured $p$ values of $+4.2 \%$ and $+1.9 \%$ (i.e. Stokes $\mathrm{U}$ is consistent with zero intensity and Stokes Q is positive) for W51_1 and W51_2, $\theta_{l o s} \gtrsim 55^{\circ}$. Unfortunately, unless a maser polarization model is created for the $\mathrm{J}=3 / 2 \mathrm{OH}(1720 \mathrm{MHz})$ transition a more quantitative analysis is not be possible.

A maser's linear polarization position angle $\chi^{\text {maser }}$ can be either parallel or perpendicular to the magnetic field in the plane-of-the-sky $B_{4}$. However, maser theories also predict that for $\theta_{\text {los }}>55^{\circ}, \chi^{\text {maser }} \perp B_{\perp}$ (see e.g. Elitzur 1998; Watson \& Wyld 2001). As mentioned above, we expect that $|\vec{B}|$ lies parallel to the plane of the shock front (perpendicular to its direction of motion), and in principle the orientation of the shock front itself can be discerned from the morphology of the shocked $\mathrm{CO}(3-2)$ emission. Although there is significant confusion from quiescent gas at the LSR velocity of the W51B molecular cloud $\left(\sim 70 \mathrm{~km} \mathrm{~s}^{-1}\right)$, the $\mathrm{CO}(3-2)$ emission from the wings of the broad component (see Fig. 8b, f) appears to be oriented EastWest. This is also apparent from the $\mathrm{CO}(3-2)$ integrated intensity image (Fig. 8 a $)$ which is dominated by the broad component. An East-West orientation for the molecular shock front is approximately perpendicular to that found for the linear polarization position angle $\chi^{\text {maser }}$ (see §3.2.1; Fig. $2 \mathrm{a}$ ). This result is in good agreement with the expectation described above based on $p$ that $\theta_{\text {los }}>55^{\circ}$ and hence we have $\chi^{\text {maser }} \perp B_{\perp}$. The situation where $\chi^{\text {maser }}$ is perpendicular to the shock front is also seen in W28 but the opposite orientation is observed for W44 (Hoffman et al. 2005a, b) . The fact that some sources fall into the $\chi^{\text {maser }} \| B_{\text {। }}$ case and some the $\chi^{\text {maser }} \perp B_{\perp}$ breaks the uncomfortable coincidence pointed out by Brogan et al. (2000) that since all SNR OH $(1720 \mathrm{MHz})$ masers have linear polarization percentages of $\sim 10 \%$ they could all have essentially the same $B \|$ which is extremely unlikely.

A final consideration is whether the observed $p$ represents the true polarized intensity. The observed polarized intensity could be a lower limit to the true intensity, owing to the factors mentioned by Brogan et al. (2000), including including Faraday depolarization and tangling of the magnetic field lines within the masing region. Those authors found that the Faraday depolarization length is only about three times larger than the estimated maser gain length of about $2 \times 10^{17} \mathrm{~cm}$ (Lockett et al. 1999). The similarity of the gain and Faraday depolarization lengths suggests that Faraday depolarization is possible, but the new data do not allow further insight on this issue. Luckily even in the presence of Faraday depolarization the net polarization P.A. is not expected to change (Elitzur 1992). We can now exclude the second possibility down to sizescales of $\sim 10^{15} \mathrm{~cm}$ (i.e. 10 mas at $6 \mathrm{kpc}$ ) based on the fact that across 4 orders of magnitude in beam area from VLA to VLBA resolution the observed Zeeman line splitting did not increase (except as consistent with spectral blending) as would be expected if the magnetic field were tangled on small size scales. 


\section{SUMMARY \& CONCLUSIONS}

We have presented a wide range of new data toward the W51C SNR and the W51B H II region complex. Using MERLIN and the VLBA we have spatially resolved the $\mathrm{OH}$ (1720 $\mathrm{MHz})$ masers in this region and explored their magnetic field properties, establishing that (1) the magnetic field strengths range from 1.5 to $2.2 \mathrm{mG}$ (using the thermal Zeeman equation); (2) the field strengths do not increase with angular resolution (except where features were spatially unresolved and spectrally blended by previous, lower-resolution observations) suggesting the field is relatively smooth on these scales; (3) the maser spots sizes are relatively large compared to $\mathrm{H}$ II region masers, with linear dimensions of $(1-4) \times 10^{15} \mathrm{~cm}$ or $\sim 60-240 \mathrm{AU}$ at $6 \mathrm{kpc}$ and brightness temperatures of $(0.4-6.0) \times 10^{9} \mathrm{~K}$; (4) the linear polarized intensities are a few percent and the position angle of the linear polarization is nearly zero; and (5) the linear polarization properties suggest that the angle between the magnetic field vector and the line-of-sight is $\gtrsim 55 \mathrm{deg}$, and that the difference between the magnetic field strengths measured using the thermal equation and the total field strength is likely to be small. More quantitative analysis requires a detailed polarization model for the $\mathrm{J}=3 / 2$ case.

We have presented the most sensitive, highest angular resolution long wavelength images of this region to date at $90 \mathrm{~cm}$ and $400 \mathrm{~cm}$ observed with the VLA showing the non-thermal radio continuum emission from the W51C SNR with unprecedented detail. These data reveal (1) the presence of non-thermal radio continuum emission in the vicinity of the $\mathrm{OH}(1720$ $\mathrm{MHz}$ ) masers, which we denote W51B_NT; (2) that the W51B H II region complex must lie in front of the W51C SNR in agreement with previous soft X-ray observations; and (3) that the nearby H II region G49.2-0.3 ( 2.3' east of the masers) has been at least partially enveloped by the W51C SNR by comparing the expected versus observed $400 \mathrm{~cm}$ absorption. Through comparison with previous X-ray data, we also find that a source of hard X-rays is coincident with W51B_NT, though the signal is too faint to model the spectrum.

Using observations of $\mathrm{CO}(3-2),{ }^{13} \mathrm{CO}(2-1), \mathrm{HCO}^{+}(3-2)$, and $\mathrm{HCN}(3-2)$ in the vicinity around the $\mathrm{OH}(1720 \mathrm{MHz})$ masers, we have discovered a ring of shocked gas partially encircling the non-thermal emission of W51B_NT, coincident spatially and kinematically with the $\mathrm{OH}(1720 \mathrm{MHz})$ masers. Radiative transfer modeling of the physical conditions in the narrow velocity, unshocked gas yields a column density of $(4-6) \times 10^{21} \mathrm{~cm}^{-2}$, a density of $(1-4) \times 10^{3} \mathrm{~cm}^{-3}$, and a temperature of $26-32 \mathrm{~K}$. The broad velocity, shocked gas is significantly smaller than the beam leading to a range of possible column densities $\left(0.3-1.5 \times 10^{23} \mathrm{~cm}^{-2}\right)$ and hence physical conditions, but the most likely density and temperatures are $1-2 \times 10^{4} \mathrm{~cm}^{-3}$ and $50-100 \mathrm{~K}$, consistent with the passage of a C-shock. 
CLB thanks the JCMT and the University of Hawaii for fellowship support during the course of this research. IMH thanks NRAO for pre-doctoral fellowship support during the course of this project. We would also like to thank Professors William (Bill) Watson and Moshe Eliztur for helpful discussions about maser polarization properties. We are also grateful for the help provided by Darek Lis and Simon Radford in obtaining the CSO spectra. This research has made use of NASA's Astrophysics Data System. We acknowledge the use of NASA's SkyView facility (http://skyview.gsfc.nasa.gov) located at NASA Goddard Space Flight Center.

\section{REFERENCES}

Abdo, A. A., Ackermann, M., Ajello, M., et al. 2009, ApJ, 706, L1

Aguirre, J. E., Ginsburg, A. G., Dunham, M. K., et al. 2011, ApJS, 192, 4

Aleksić, J., Alvarez, E. A., Antonelli, L. A., et al. 2012, A\&A, 541, A13

Benjamin, R. A., et al. 2005, ApJ, 630, L149

Bieging, J. H., Peters, W. L., \& Kang, M. 2010, ApJS, 191, 232

Brogan, C. L., Lazio, T. J., Kassim, N. E., \& Dyer, K. K. 2005, AJ, 130, 148

Brogan, C. L., Devine, K. E., Lazio, T. J., Kassim, N. E., Tam, C. R., Brisken, W. F., Dyer, K. K., \& Roberts, M. S. E. 2004, AJ, 127, 355

Brogan, C. L., Frail, D. A., Goss, W. M., \& Troland, T. H. 2000, ApJ, 537, 875

Castro, D., \& Slane, P. 2010, ApJ, 717, 372

Ceccarelli, C., Hily-Blant, P., Montmerle, T., et al. 2011, ApJ, 740, L4

Claussen, M. J., Goss, W. M., Desai, K. M., \& Brogan, C. L. 2002, ApJ, 580, 909

Claussen, M. J., Goss, W. M., Frail, D. A., \& Desai, K. 1999, ApJ, 522, 349

Claussen, M. J., Goss, W. M., Frail, D. A., \& Seta, M. 1999, AJ, 117, 1387

Claussen, M. J., Frail, D. A., Goss, W. M., \& Gaume, R. A. 1997, ApJ, 489, 143

Copetti, M. V. F., \& Schmidt, A. A. 1991, MNRAS, 250, 127 
Doty, S. D., van Dishoeck, E. F., van der Tak, F. F. S., \& Boonman, A. M. S. 2002, A\&A, 389,446

Elitzur, M. 1976, ApJ, 203, 124

Elitzur, M. 1998, ApJ, 504, 390

Elitzur, M. 1996, ApJ, 457, 415

Elitzur, M. 1992, Astrophysics and Space Science Library, 170,

Frail, D. A. 2011, Mem. Soc. Astron. Italiana, 82, 703

Frail, D. A., Goss, W. M., Reynoso, E. M., et al. 1996, AJ, 111, 1651

Frail, D. A., \& Mitchell, G. F. 1998, ApJ, 508, 690

Green, A. J., Frail, D. A., Goss, W. M., \& Otrupcek, R. 1997, AJ, 114, 2058

Han, S. W. 2001, Master's thesis, Seoul National University.

Hanabata, Y., Sawada, M., Katagiri, H., Bamba, A., \& Fukazawa, Y. 2012, arXiv:1212.1993

Hewitt, J. W., Yusef-Zadeh, F.\& Wardle, M. 2008, ApJ, 683, 189

Hewitt, J. W., \& Yusef-Zadeh, F. 2009, ApJ, 694, L16

Hewitt, J. W., Yusef-Zadeh, F.\& Wardle, M. 2009, ApJ, 706, L270

Hoffman, I. M., Goss, W. M., Brogan, C. L., \& Claussen, M. J. 2005, ApJ, 627, 803

Hoffman, I. M., Goss, W. M., Brogan, C. L., \& Claussen, M. J. 2005, ApJ, 620, 257

Hoffman, I. M., Goss, W. M., Brogan, C. L., Claussen, M. J., \& Richards, A. M. S. 2003, ApJ, 583, 272

Jiang, B., Chen, Y., Wang, J., et al. 2010, ApJ, 712, 1147

Kolpak, M. A., Jackson, J. M., Bania, T. M., Clemens, D. P., \& Dickey, J. M. 2003, ApJ, 582,756

Koo, B.-C. 1997, ApJS, 108, 489

Koo, B.-C., \& Moon, D.-S. 1997, ApJ, 485, 263

Koo, B.-C. 1999, ApJ, 518, 760 
Koo, B.-C., Lee, J.-J., \& Seward, F. D. 2002, AJ, 123, 1629

Koo, B.-C., Lee, J.-J., Seward, F. D., \& Moon, D.-S. 2005, ApJ, 633, 946

Koo, B.-C., Kim, K.-T., \& Seward, F. D. 1995, ApJ, 447, 211

Koralesky, B., Frail, D. A., Goss, W. M., Claussen, M. J., \& Green, A. J. 1998, AJ, 116, 1323

Lazendic, J.-S., Wardle, M. , Whiteoak, J. -B. , Burton, M.-G. , \& Green, A.J. 2010, MNRAS, 409, 371

Lockett, P., Gauthier, E., \& Elitzur, M. 1999, ApJ, 511, 235

Lockman, F. J. 1989, ApJS, 71, 469

McDonnell, K. E., Wardle, M., \& Vaughan, A. E. 2008, MNRAS, 390, 49

Méhault, J. 2011, Mem. Soc. Astron. Italiana, 82, 756

Milam, S. N., Savage, C., Brewster, M. A., Ziurys, L. M., \& Wyckoff, S. 2005, ApJ, 634, 1126

Minier, V., Booth, R. S., \& Conway, J. E. 2002, A\&A, 383, 614

Moisés, A. P., Damineli, A., Figuerêdo, E., et al. 2011, MNRAS, 411, 705

Moon, D.-S., \& Koo, B.-C. 1994, Journal of Korean Astronomical Society, 27, 81

Nord, M. E., Henning, P. A., Rand, R. J., Lazio, T. J. W., \& Kassim, N. E. 2006, AJ, 132, 242

Ossenkopf, V., \& Henning, T. 1994, A\&A, 291, 943

Pavlakis, K. G., \& Kylafis, N. D. 1996, ApJ, 467, 300

Pihlström, Y. -M. ,Sjouwerman, L. -O. ,\& Mesler, R. -A. 2011, ApJ, 740, 66

Reach, W. T., Rho, J., \& Jarrett, T. H. 2005, ApJ, 618, 297

Reach, W. T., Rho, J., Jarrett, T. H., \& Lagage, P.-O. 2002, ApJ, 564, 302

Richards, A. M. S., Elitzur, M., \& Yates, J. A. 2011, A\&A, 525, A56

Sato, M., Reid, M. J., Brunthaler, A., \& Menten, K. M. 2010, ApJ, 720, 1055 
Schöier, F. L., van der Tak, F. F. S., van Dishoeck, E. F., \& Black, J. H. 2005, A\&A, 432, 369

Sjouwerman, L. O., \& Pihlström, Y. M. 2008, ApJ, 681, 1287

Subrahmanyan, R., \& Goss, W. M. 1995, MNRAS, 275, 755

van der Tak, F. F. S., Black, J. H., Schöier, F. L., Jansen, D. J., \& van Dishoeck, E. F. 2007, A\&A, 468, 627

Uchiyama, Y., Funk, S., Katagiri, H., et al. 2012, ApJ, 749, L35

Velusamy, T., \& Kundu, M. R. 1974, A\&A, 32, 375

Wardle, M. 1999, ApJ, 525, L101

Wardle, M., \& Yusef-Zadeh, F. 2002, Science, 296, 2350

Watson, W. D., \& Wyld, H. W. 2001, ApJ, 558, L55

Woodall, J. M., \& Gray, M. D. 2007, MNRAS, 378, L20

Yusef-Zadeh, F., Roberts, D. A., Goss, W. M., Frail, D. A., \& Green, A. J. 1996, ApJ, 466, L25

This preprint was prepared with the AAS IATEX macros v5.2. 
Table 1. Observing Parameters

\begin{tabular}{|c|c|}
\hline Parameter & Value \\
\hline \multicolumn{2}{|c|}{ MERLIN OH (1720MHz) masers } \\
\hline Date & 2002 Jan 14, 15, 18 (MMO1B06) \\
\hline Bandwidth & $0.25 \mathrm{MHz}$ \\
\hline Spectral channels & 256 \\
\hline Channel separation & $0.17 \mathrm{~km} \mathrm{~s}^{-1}$ \\
\hline Velocity resolution & $0.24 \mathrm{~km} \mathrm{~s}^{-1}$ \\
\hline Spectral line rms noise ${ }^{a}$ & $5 \mathrm{mJy}^{\text {beam }^{-1}}$ \\
\hline Synthesized beam & 221 mas $\times 125$ mas P.A. $=22.8^{\circ}$ \\
\hline \multicolumn{2}{|c|}{ VLBA OH $(1720 \mathrm{MHz})$ masers } \\
\hline Dates & 2000 Dec 02,03, \& 04 (BB129) \\
\hline Bandwidth & $0.25 \mathrm{MHz}$ \\
\hline Spectral channels & 256 \\
\hline Channel separation & $0.17 \mathrm{~km} \mathrm{~s}^{-1}$ \\
\hline Velocity resolution & $0.24 \mathrm{~km} \mathrm{~s}^{-1}$ \\
\hline Spectral line rms noise ${ }^{a}$ & 9 mJy beam $^{-1}$ \\
\hline Synthesized beam & $12.5 \operatorname{mas} \times 6.3$ mas P.A. $=-5.2^{\circ}$ \\
\hline \multicolumn{2}{|c|}{ VLA $400 \mathrm{~cm}$ continuum } \\
\hline Dates B-array & 2002 Jun 06 \& 22 (AB1031) \\
\hline Date C-array & 2006 Oct $24(\mathrm{AB} 1219)$ \\
\hline Bandwidth & $1.5 \mathrm{MHz}$ \\
\hline Spectral Channels & 64 \\
\hline Continuum rms noise & 100 mJy beam ${ }^{-1}$ \\
\hline Synthesized beam $(\mathrm{B}+\mathrm{C})$ & $92^{\prime \prime} .2 \times 83^{\prime \prime} .5$ P.A. $29.8^{\circ}$ \\
\hline \multicolumn{2}{|c|}{ VLA $90 \mathrm{~cm}$ continuum } \\
\hline Date A-array & 2003 Aug 23 (AB1089) \\
\hline Dates B-array & 2002 Jun 06 \& 22(AB1031) \\
\hline Dates C-array & 2002 Nov $02 ; 2002$ Dec $13 \& 30($ AB1031) \\
\hline Date D-array & 2003 Feb 08 (AB1077) \\
\hline Bandwidth & $3.0 \mathrm{MHz}$ \\
\hline
\end{tabular}


Table 1-Continued

\begin{tabular}{ll}
\hline \hline \multicolumn{1}{c}{ Parameter } & \multicolumn{1}{c}{ Value } \\
\hline Spectral Channels & 32 \\
Continuum rms noise & $12 \mathrm{mJy} \mathrm{beam}^{-1}$ \\
Synthesized beam (B+C+D) & $34^{\prime \prime} 8 \times 32^{\prime \prime} 9$ P.A. $=-86^{\circ}$ \\
Synthesized beam (A+B+C+D) & $20^{\prime \prime} .7 \times 19^{\prime \prime} 8$ P.A. $=44^{\circ}$ \\
\hline
\end{tabular}

VLA $21 \mathrm{~cm}$ continuum and $\mathrm{H}_{\mathrm{I}}$

Date C-array

2006 Nov 13 (AB1219)

Date D-array (used for continuum only) $1992 \mathrm{Jul}$ (AK301)

Bandwidth (C)

$3.0 \mathrm{MHz}$

Spectral Channels $(\mathrm{C})$

32

Continuum rms noise $(\mathrm{C}+\mathrm{D})$

5 mJy beam ${ }^{-1}$

$3 \mathrm{mJy}$ beam $^{-1}$

Spectral Line rms noise ${ }^{a}(\mathrm{C})$

$34^{\prime \prime} .8 \times 32^{\prime \prime} .9$ P.A. $=-86^{\circ}$

Synthesized beam $(\mathrm{C}+\mathrm{D}$ continuum)

$14^{\prime \prime} 7 \times 14^{\prime \prime} .5$ P.A. $=14^{\circ}$

JCMT $345 \mathrm{GHz} \mathrm{CO}(3-2)$

Dates

Rest frequency

2004 Mar 29; Apr 20-25; Jun 11 \& 12 (m04ah45a2)

Observing mode

$345.79599 \mathrm{GHz}$

Velocity resolution

Single sideband

$1.08 \mathrm{~km} \mathrm{~s}^{-1}$

Primary Beam

$14^{\prime \prime}$

Spectral line rms noise ${ }^{a}$

$0.1 \mathrm{~K}$

JCMT $230 \mathrm{GHz}{ }^{13} \mathrm{CO}(2-1)$

\section{Dates}

Rest frequency

Observing mode

Velocity resolution

Primary Beam

Spectral line rms noise ${ }^{a}$
2005 Sep 22 (m05bh49c)

$220.39868 \mathrm{GHz}$

Double sideband

$1.1 \mathrm{~km} \mathrm{~s}^{-1}$

$20^{\prime \prime}$

$0.15 \mathrm{~K}$

$\mathrm{CSO} \mathrm{HCO}+(3-2) \& \mathrm{HCN}(3-2)$ 
Table 1-Continued

\begin{tabular}{ll}
\hline \hline \multicolumn{1}{c}{ Parameter } & \multicolumn{1}{c}{ Value } \\
\hline Rest frequencies & 265.886 \& 267.558 GHz \\
Observing mode & Double sideband \\
Velocity resolution & $0.6 \& 1.2 \mathrm{~km} \mathrm{~s}^{-1}$ \\
Primary Beam & $26^{\prime \prime}$ \\
Spectral line rms noise $^{a}$ & $0.02 \& 0.014 \mathrm{~K}$ \\
\hline
\end{tabular}

aThe rms noise per channel measured in main beam temperature $T_{M B}$.

${ }^{\mathrm{b}}$ Project codes are shown after the observation date(s).

Table 2. Maser Spectral Line Parameters

\begin{tabular}{|c|c|c|c|c|c|c|c|c|}
\hline Maser Spot & Telescope & $\alpha\left(\begin{array}{lll}\mathrm{h} & \mathrm{J} 2000 \mathrm{C} & \mathrm{s}\end{array}\right)$ & $\begin{array}{l}\text { ordinates }{ }^{\mathrm{b}} \\
\delta \delta\left({ }^{\circ},{ }^{\prime \prime}\right)\end{array}$ & $\begin{array}{l}V_{L S R}(\text { peak }) \\
\left(\mathrm{km} \mathrm{s}^{-1}\right)\end{array}$ & $\begin{array}{c}\Delta V_{F W H M}(\text { peak }) \\
\left(\mathrm{km} \mathrm{s}^{-1}\right)\end{array}$ & $\begin{array}{c}\int S d v(\text { peak }) \\
\left(\text { Jy beam }^{-1 *} \mathrm{~km} \mathrm{~s}^{-1}\right)\end{array}$ & $\begin{array}{c}\int S d v(i n t) \\
\left(\mathrm{Jy}^{*} \mathrm{~km} \mathrm{~s}^{-1}\right)\end{array}$ & $\begin{array}{c}B \\
(\mathrm{mG})\end{array}$ \\
\hline \multirow{3}{*}{ W51_1 } & $\mathrm{VLA}^{\mathrm{a}}$ & & & $72.0(0.02)$ & $0.9(0.05)$ & $2.6(0.05)$ & $2.6(0.05)$ & $1.5 \pm 0.05$ \\
\hline & MERLIN & & & $72.018(0.002)$ & $0.902(0.004)$ & $1.234(0.004)$ & $1.38(0.02)$ & $1.5 \pm 0.05$ \\
\hline & VLBA & 192253.8210 & +141543.462 & $72.06(0.01)$ & $0.901(0.03)$ & $0.211(0.007)$ & $1.05(0.05)$ & $1.5 \pm 0.2$ \\
\hline \multirow[t]{4}{*}{ W51_2 } & $\mathrm{VLA}^{\mathrm{a}}$ & & & $69.1(0.02)$ & $1.2(0.05)$ & $6.1(0.05)$ & $6.1(0.05)$ & $1.9 \pm 0.1$ \\
\hline & MERLIN & & & $69.031(0.001)$ & $1.183(0.002)$ & $2.939(0.004)$ & $3.12(0.02)$ & $1.9 \pm 0.05$ \\
\hline & VLBA_a & 192254.3632 & +141540.219 & $68.965(0.002)$ & $1.064(0.004)$ & $1.029(0.004)$ & $2.07(0.02)$ & $1.7 \pm 0.1$ \\
\hline & VLBA_b & 192254.3608 & +141540.247 & $69.050(0.004)$ & $0.957(0.009)$ & $0.700(0.005)$ & $1.20(0.04)$ & $2.2 \pm 0.1$ \\
\hline
\end{tabular}

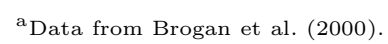

${ }^{\mathrm{b}}$ Position Uncertainties are $\sim 1$ mas. Only the most accurate VLBA positions are given, the VLA and MERLIN positions agree to within their individual (larger) uncertainties. 
Table 3. Fitted Parameters of Thermal Molecular Lines Toward OH Masers

\begin{tabular}{ccccc}
\hline \hline Transition $^{\mathrm{a}}$ & Component & $\begin{array}{c}\text { Peak v } \\
\left(\mathrm{km} \mathrm{s}^{-1}\right)\end{array}$ & $\begin{array}{c}\text { Peak TMB } \\
(\mathrm{K})\end{array}$ & $\begin{array}{c}\text { FWHM } \\
\left(\mathrm{km} \mathrm{s}^{-1}\right)\end{array}$ \\
\hline${ }^{12} \mathrm{CO} \mathrm{3-2}$ & narrow & $70.65 \pm 0.03$ & $18.57 \pm 0.28$ & $4.99 \pm 0.09$ \\
& broad & $70.12 \pm 0.18$ & $6.72 \pm 0.26$ & $18.64 \pm 0.52$ \\
& blueshifted & $59.40 \pm 0.15$ & $2.30 \pm 0.26$ & $3.00 \pm 0.43$ \\
\hline${ }^{13}$ CO 2-1 & narrow & $71.05 \pm 0.07$ & $4.31 \pm 0.22$ & $3.20 \pm 0.20$ \\
& broad & $70.1^{\mathrm{a}}$ & $0.49 \pm 0.16$ & $15.47 \pm 4.43$ \\
& blueshifted & $59.4^{\mathrm{b}}$ & $0.43 \pm 0.18$ & $3.0^{\mathrm{b}}$ \\
\hline HCO+3-2 & narrow & $71.03 \pm 0.16$ & $0.20 \pm 0.02$ & $4.4 \pm 0.5$ \\
& broad & $69.6 \pm 0.3$ & $0.21 \pm 0.02$ & $18.0 \pm 1.0$ \\
& blueshifted & $59.3 \pm 0.3$ & $0.07 \pm 0.01$ & $3.0^{\mathrm{b}}$ \\
\hline HCN 3-2 & narrow & $69.6 \pm 0.2$ & $0.07 \pm 0.01$ & $1.7 \pm 0.4$ \\
& broad & $69.7 \pm 0.3$ & $0.16 \pm 0.02$ & $14.9 \pm 1.0$ \\
& blueshifted & $57.4 \pm 0.4$ & $0.06 \pm 0.01$ & $3.0^{\mathrm{b}}$ \\
\hline
\end{tabular}

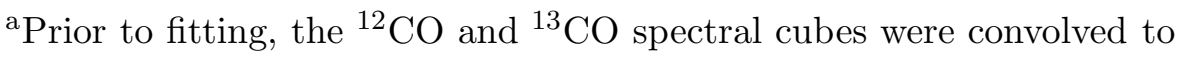
a beamsize of $26^{\prime \prime}$ in order to match the CSO $\mathrm{HCO}+$ spectrum. These fitted values were used in the modeling described in $\S 4.1$.

${ }^{b}$ Values without uncertainties were fixed during the fit. 